\title{
Hepatic ketogenic insufficiency reprograms hepatic glycogen metabolism and the lipidome
}

D. André d'Avignon, ${ }^{1,2}$ Patrycja Puchalska, ${ }^{1,2}$ Baris Ercal,, ${ }^{2,3}$ YingJu Chang, ${ }^{1,2}$ Shannon E. Martin, ${ }^{2,4}$ Mark J. Graham, ${ }^{5}$ Gary J. Patti, ${ }^{6}$ Xianlin Han, ${ }^{2,7}$ and Peter A. Crawford ${ }^{1,2,8}$

'Division of Molecular Medicine, Department of Medicine, University of Minnesota, Minneapolis, Minnesota, USA. ${ }^{2}$ Center for Metabolic Origins of Disease, Sanford Burnham Prebys Medical Discovery Institute, Orlando, Florida, USA. ${ }^{3}$ Department of Psychiatry, Washington University, St. Louis, Missouri, USA. ${ }^{4}$ Pathobiology Graduate Program, Brown University, Providence, Rhode Island, USA. ${ }^{5}$ Ionis Pharmaceuticals, Carlsbad, California, USA. ${ }^{6}$ Department of Chemistry, Washington University, St. Louis, Missouri, USA. Barshop Institute for Longevity and Aging Studies, Department of Medicine, Division of Diabetes, University of Texas Health Science Center at San Antonio, San Antonio, Texas, USA. ${ }^{8}$ Department of Biochemistry, Molecular Biology, and Biophysics, University of Minnesota, Minneapolis, Minnesota, USA

While several molecular targets are under consideration, mechanistic underpinnings of the transition from uncomplicated nonalcoholic fatty liver disease (NAFLD) to nonalcoholic steatohepatitis (NASH) remain unresolved. Here we apply multiscale chemical profiling technologies to mouse models of deranged hepatic ketogenesis to uncover potential NAFLD driver signatures. Use of stable-isotope tracers, quantitatively tracked by nuclear magnetic resonance (NMR) spectroscopy, supported previous observations that livers of wild-type mice maintained long term on a high-fat diet (HFD) exhibit a marked increase in hepatic energy charge. Fed-state ketogenesis rates increased nearly 3 -fold in livers of HFD-fed mice, a greater proportionate increase than that observed for tricarboxylic acid (TCA) cycle flux, but both of these contributors to overall hepatic energy homeostasis fueled markedly increased hepatic glucose production (HCP). Thus, to selectively determine the role of the ketogenic conduit on HCP and oxidative hepatic fluxes, we studied a ketogenesis-insufficient mouse model generated by knockdown of the mitochondrial isoform of 3-hydroxymethylglutaryl-CoA synthase (HMCCS2). In response to ketogenic insufficiency, TCA cycle flux in the fed state doubled and HCP increased more than $60 \%$, sourced by a 3-fold increase in glycogenolysis. Finally, high-resolution untargeted metabolomics and shotgun lipidomics performed using ketogenesis-insufficient livers in the fed state revealed accumulation of bis(monoacylglycero)phosphates, which also accumulated in livers of other models commonly used to study NAFLD. In summary, natural and interventional variations in ketogenesis in the fed state strongly influence hepatic energy homeostasis, glucose metabolism, and the lipidome. Importantly, HCP remains tightly linked to overall hepatic energy charge, which includes both terminal fat oxidation through the TCA cycle and partial oxidation via ketogenesis.

Conflict of interest: MJG is an employee and shareholder of lonis Pharmaceuticals Inc.

Submitted: January 11, 2018

Accepted: May 17, 2018

Published: June 21, 2018

Reference information: JCI Insight. 2018;3(12):e99762. https://doi.org/10.1172/jci. insight.99762.

\section{Introduction}

Among its multitude of functions, the heathy liver produces the primary fuel glucose and the alternative fuel ketone bodies to furnish peripheral organs with nutrients needed to meet energy requirements. Longterm overnutrition, common with Western-style diets, often provokes the development of nonalcoholic fatty liver disease (NAFLD), a condition affecting 30\% of adults in the United States in which ectopic fat accumulates in otherwise normal hepatocytes, and hepatic glucose production (HGP) becomes less responsive to the inhibitory effects of insulin (1-5). Through incompletely understood mechanisms, as high as $10 \%$ of those with NAFLD progress to nonalcoholic steatohepatitis (NASH), a condition characterized by hepatocyte injury, hepatic inflammation, and fibrosis, which predisposes individuals to cardiovascular disease, and can progress further to cirrhosis and hepatocellular carcinoma (1). Understanding disease pathogenesis and markers for the progression NAFLD to NASH represent critical areas of focus in efforts to counter the developing healthcare crisis stemming from this epidemic. 
As a fat oxidation spillover pathway most actively engaged in carbohydrate-restricted settings, ketogenesis is not commonly considered among the influences of NAFLD pathogenesis. Normally during the postabsorptive state, nonesterified fatty acids (NEFAs) generated by adipose tissue lipolysis are proportionally catabolized within the mitochondrion through $\beta$-oxidation, generating acetyl-CoA. In hepatocytes, mitochondrial $\beta$-oxidation-derived acetyl-CoA is either terminally oxidized via the tricarboxylic acid (TCA) cycle or diverted to ketogenesis for generation of the energy-rich ketone bodies acetoacetate (AcAc) and $D-\beta$-hydroxybutryate $(\beta \mathrm{OHB})$ for export. Ketone bodies are generated from fatty acid-derived acetyl-CoA through a series of reactions requiring the fate-committing mitochondrial isoform of 3-hydroxymethylglutaryl (HMG)-CoA synthase (HMGCS2) that generates HMG-CoA, which is converted to AcAc by mitochondrial HMG-CoA lyase (HMGCL). AcAc is reduced to $\beta \mathrm{OHB}$ by the mitochondrial and $\mathrm{NAD}^{+} /$ $\mathrm{NADH}-$ dependent enzyme $\beta \mathrm{OHB}$ dehydrogenase (BDH1). Ketogenesis is relatively restricted to hepatocytes due to selective HMGCS2 in these cells, and while the reaction catalyzed by HMGCL is also required for ketogenesis, it is not uniquely fate-committing because the HMGCL step is downstream of that catalyzed by HMGCS2, and HMGCL is also indispensable for leucine catabolism, in which HMGCS2 does not play a role (6). In the cytoplasm, the HMGCS1 isoform catalyzes the same condensation reaction as that catalyzed by HMGCS2 in mitochondria, and the former is a key enzyme in the mevalonate biosynthetic pathway, preceding the step catalyzed by HMG-CoA reductase. After export from mitochondria and from hepatocytes into the circulation, ketone bodies are highly efficient substrates for extrahepatic tissues with the potential to support up to $20 \%$ of total human energy expenditure (7-9).

While playing a relatively minor role in the normal fed state, the mitochondrial ketogenic conduit may be an important consideration in NAFLD because a hallmark of NAFLD is an abundance of fat extracted by the liver that is mismatched to mitochondrial capability to terminally dispose it. Lipolytic excess, coupled with unchecked hepatic lipogenesis, leads not only to ectopic fat accumulation in the hepatocyte, but places an excessive fat-processing burden on the hepatic mitochondrion (10). Increased oxidative flux through the TCA cycle has been associated with NAFLD (10-13). Increased TCA cycle flux provides a terminal fate for a portion of excess fat but does not fully compensate for the load delivered to the hepatocyte, and TCA cycle flux increases also predispose to oxidative stress. By disposing a product of hepatic $\beta$-oxidation, acetyl-CoA, ketogenesis prevents the need for hepatic terminal fat oxidation, offloading a substantial fraction of reducing equivalents from the liver to extrahepatic tissues, and thus ketogenesis could be an ideal pathway to clear excess lipid. In spite of this, ketogenesis is underutilized in prediabetic obesity, in that through both fed and fasting states, ketogenesis is inadequately tuned to available fat, and is often diminished relative to lean controls (12-18). Many of these observations were collected under fasting conditions in which ketogenesis is most active, but ketogenesis during nonfasting periods is quite variable, and could be an under-utilized fat disposal conduit, attenuated by metabolic and hormonal control of HMGCS2 (6).

Recently, analysis of mice with HMGCS2 deficiency in the carbohydrate-laden, high-fat diet-fed (HFD-fed) state revealed that ketogenic insufficiency could model the evolution of uncomplicated NAFLD to NASH (19). Here, we apply dual isotope tracer nuclear magnetic resonance (NMR) spectroscopy, isotope-label-tracking untargeted metabolomics using high-resolution liquid chromatography/mass spectrometry (LC/MS), and shotgun lipidomics studies of this ketogenesis-insufficient mouse model in the carbohydrate-laden fed state, prior to the onset of injury and inflammation, to reveal significant derangements in glucose, TCA cycle, and lipid metabolism incurred by variations of ketogenic capacity.

\section{Results}

Long-term HFD increases central hepatic metabolic fluxes. We and others have posed that ketogenesis in livers of obese humans and rodents insufficiently matches the fat delivered to it (6). To formally quantify metabolic fluxes in livers of mice with metabolic features of NAFLD, we fed wild-type C57BL/6 mice a long-term (40-week-old male mice, 35 weeks on $60 \% \mathrm{kcal}$ fat diet) HFD, comparing them to age-matched chow-fed controls. As expected, greater than 70\% increase in body weight, compared with controls, and histological evidence of profound steatosis were uniquely observed in the HFD-fed animals (Supplemental Figure 1, A-C; supplemental material available online with this article; https://doi.org/10.1172/jci. insight.99762DS1). We then performed 60-minute portal vein perfusions of mice from both groups in the fed state, using oxygenated Krebs-Henseleit buffer (19-21) lacking glucose, but containing unlabeled $0.2 \mathrm{mM}$ sodium octanoate (exogenous fat source), $3 \%{ }^{2} \mathrm{H}_{2} \mathrm{O}$, and $0.2 \mathrm{mM}$ sodium $\left[\mathrm{U}-{ }^{13} \mathrm{C}\right]$ propionate, and collected the hepatic venous effluent. After metabolite extraction and quantification using ${ }^{1} \mathrm{H}-\mathrm{NMR}$ (Supplemental 
A

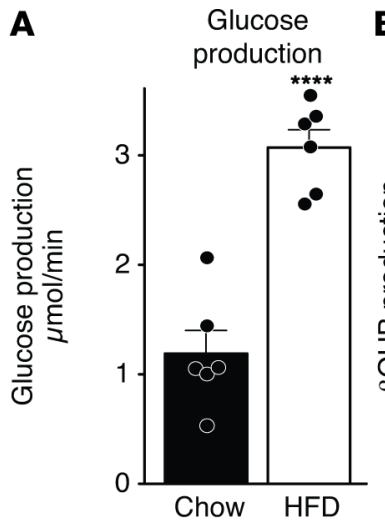

D

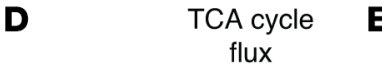

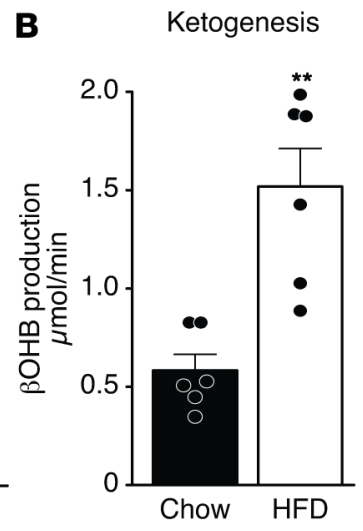

\section{$\mathbf{E}$}

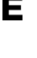

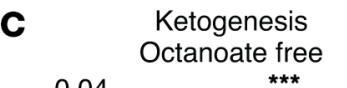

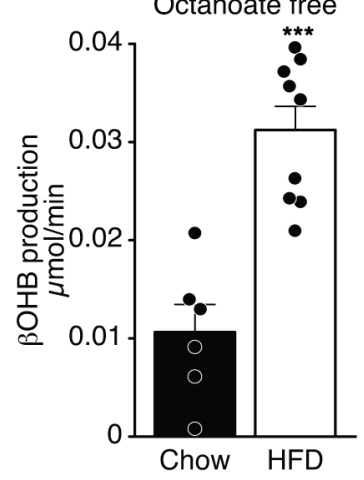

$\mathbf{F}$

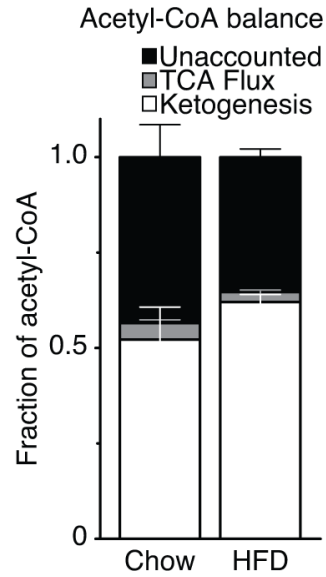

Figure 1. Increased hepatic oxidative fluxes provoked by sustained high-fat diet feeding in wild-type mice. (A) Glucose production and (B) $\beta O H B$ production by livers of age-matched mice fed standard chow versus $60 \%$ kcal fat diet for 35 weeks, quantified by ${ }^{1} \mathrm{H}-\mathrm{NMR}$ of hepatic venous effluent from 60 -minute portal vein perfusion using an oxygenated buffer containing 0.2 $\mathrm{mM}$ unlabeled sodium octanoate as exogenous fat, $3 \%{ }^{2} \mathrm{H}_{2} \mathrm{O}$, and $0.2 \mathrm{mM}$ sodium [U- ${ }^{13} \mathrm{C}$ ]propionate. (C) $\beta \mathrm{OHB}$ production rates in separate perfusions using an oxygenated buffer that was otherwise identical but lacked an exogenous fat source. (D) TCA cycle flux rates, quantified by ${ }^{13} \mathrm{C}$-NMR of monoacetone $\left[{ }^{13} \mathrm{C} /{ }^{2} \mathrm{H}\right]$ glucose derivatized from hepatic venous effluent ${ }^{13} \mathrm{C} /{ }^{2} \mathrm{H}$-labeled glucose, from perfusions using an oxygenated buffer containing $0.2 \mathrm{mM}$ unlabeled sodium octanoate, $3 \%{ }^{2} \mathrm{H}_{2} \mathrm{O}$, and $0.2 \mathrm{mM}$ sodium [U- ${ }^{13} \mathrm{C}$ ]propionate. (E) Octanoate consumption rates by livers of mice described and analyzed as in $\mathbf{A}$ and $\mathbf{B}$. (F) Imputed balances of octanoate-derived acetyl-CoA fates, based on octanoate consumption and measured disposal of acetyl-CoA through the TCA cycle and $\beta O H B$ production. Note that little octanoate is esterified to glycerol, and much of the unaccounted fraction is attributable to AcAc. HFD, high-fat diet. $n=6$ /group. ${ }^{* *} P<0.01$; ${ }^{* * *} P<0.001 ;{ }^{* * *} P<0.0001$. ns, not significant by Student's $t$ test.

Figure 2A), the hepatic ${ }^{13} \mathrm{C} /{ }^{2} \mathrm{H}-$ labeled glucose pool was derivatized to monoacetone glucose, from which ${ }^{2} \mathrm{H}$ and ${ }^{13} \mathrm{C}$ signals were collected, allowing hepatic oxidative fluxes to be quantified (Supplemental Figure 2, $\mathrm{B}$ and C) (10, 22). Total HGP by livers of HFD-fed mice was increased nearly 3-fold (3.07 $\pm 0.16 \mu \mathrm{mole} / \mathrm{min}$ vs. $1.19 \pm 0.21 \mu \mathrm{mole} / \mathrm{min}$ tissue in controls, $n=6$ /group, $P<0.001$; Figure $1 \mathrm{~A}$ ). All 3 contributors to HGP (glycogenolysis, glycerol, and phosphoenolpyruvate [PEP]) were increased in livers of HFD-fed mice, such that relative contributions of each to all of HGP were normal (Supplemental Figure 3, A-D). Moreover, ketogenesis was similarly increased $(1.52 \pm 0.19 \mu \mathrm{mole} / \mathrm{min}$ vs. $0.58 \pm 0.08 \mu \mathrm{mole} / \mathrm{min}$ tissue in controls, $n=6$ /group, $P=0.001$; Figure $1 \mathrm{~B}$ ), similar to previous observations in HFD-fed rodents (10). Interestingly, perfusions performed in the absence of octanoate in the perfusion buffer revealed that endogenous liver fat alone confers a 3-fold increase in ketogenesis for livers of HFD-fed animals $(0.032 \pm 0.003 \mu \mathrm{mole} / \mathrm{min}$ vs. $0.011 \pm 0.003 \mu \mathrm{mole} / \mathrm{min}$ tissue in controls, $n=6-9 /$ group, $P=0.001$; Figure $1 \mathrm{C})$. TCA cycle flux was modestly but not significantly increased in octanoate-containing perfusions of mice maintained on the HFD, compared with the chow diet cohort (Figure 1D). Nonetheless, livers of HFD-fed animals were indeed in a high-energy state, as gluconeogenesis, pyruvate cycling, and anaplerosis, in absolute terms and normalized to TCA flux, were all increased in livers of HFD-fed mice (Supplemental Figure 3, C-F), and octanoate consumption was increased by more than 2 -fold in livers of HFD-fed mice $(1.21 \pm 0.16 \mu \mathrm{mole} / \mathrm{min}$ vs. 0.56 $\pm 0.15 \mu \mathrm{mole} / \mathrm{min}$ tissue in controls, $n=6$ /group, $P=0.005$; Figure $1 \mathrm{E}$ ).

As $\beta$-oxidation of octanoate yields 4 acetyl-CoA units, we imputed the relative allocations of octanoatederived acetyl-CoA. While terminal oxidation through the TCA cycle represents a minor fate $(\leq 5 \%)$ for the carbon available from octanoate-derived acetyl-CoA in either diet setting in 40-week-old mice, ketogenesis is the major carbon consumer and energy conduit for both HFD-fed (64\%) and chow-fed (53\%) mice (Figure $1 F)$. Much of the unaccounted fates for octanoate likely includes AcAc (which is not quantified using this analytical approach), but acylcarnitines and other incompletely oxidized intermediates also comprise the balance of carbon consumption from $\beta$-oxidation, as esterification and storage of the medium-chain fatty acid octanoate is minimal (23). Notably, while the livers of HFD-fed mice exhibited 2-fold greater octanoate uptake, the corresponding fractional component dedicated to ketogenesis was only approximately $20 \%$ 
greater than that observed for the control group. Therefore, while insufficient to clear the lipid load extracted and stored, ketogenesis in obese, HFD-fed wild-type mice is increased in absolute terms, from either exogenous or endogenous fat.

Ketogenic insufficiency reprograms central hepatic glucose metabolism. Given these observations, we hypothesized that ketogenesis may be a dynamically regulated metabolic node, which can feed back to modulate normal hepatic glucose and oxidative metabolic homeostasis. To determine the effects of genetically engineered ketogenic insufficiency, we studied Hmgcs2 antisense oligonucleotide-treated (Hmgcs2-ASO-treated) and control-ASO-treated standard-chow-fed, 12- to 16-week-old male mice, 6-8 weeks after initiation of ASO knockdown. This approach results in greater than $90 \%$ loss of hepatic HMGCS2 protein within 3 weeks of ASO initiation, and HFD-induced hepatocellular injury and inflammation (19). Importantly, however, standard-chow-fed Hmgcs2-ASO-treated mice do not exhibit hepatocellular injury or inflammation. Therefore, to develop signatures that precede and thus prospectively contribute to metabolically driven hepatitis disease progression, we performed chemical profiling studies using these mice in the standardchow-fed state. To formally quantify central metabolic fluxes in the livers of these animals, we first performed 60-minute portal vein perfusions using oxygenated glucose-free buffer containing unlabeled $0.2 \mathrm{mM}$ sodium octanoate and $3 \%{ }^{2} \mathrm{H}_{2} \mathrm{O}$. Both sodium $\left[\mathrm{U}_{-}{ }^{13} \mathrm{C}\right]$ propionate-containing $(0.2 \mathrm{mM})$ and propionate-free perfusions were performed independently to confirm the absence of effect of this propionate concentration on HGP, its contributing pathways, or ketogenesis (24). Consistent with our published observations using alternative methods in the same model, ketogenesis was attenuated 10-fold in livers of Hmgcs2-ASO-treated mice (in the presence of propionate, $0.03 \pm 0.00 \mu \mathrm{mole} / \mathrm{min} / \mathrm{g}$ vs. $0.36 \pm 0.07 \mu \mathrm{mole} / \mathrm{min} / \mathrm{g}$ tissue in Hmgcs 2- and control-ASO-treated mice, respectively, $n=10-13$ /group, $P<0.001$; Figure 2A). As expected, although ketogenesis was still measurable, exclusion of the exogenous octanoate fat source in the perfusion buffer diminished ketogenesis by nearly 20 -fold in control animals, and ketogenesis derived from endogenous fat was undetectable by NMR in livers of ketogenesis-insufficient animals (Figure 2B). Excluding 0.2 $\mathrm{mM}\left[{ }^{13} \mathrm{C}\right]$ propionate had no significant effect on octanoate-supported ketogenesis in either animal cohort $(0.04 \pm 0.01 \mu \mathrm{mole} / \mathrm{min} / \mathrm{g}$ vs. $0.53 \pm 0.09 \mu \mathrm{mole} / \mathrm{min} / \mathrm{g}$ tissue in Hmgcs2- and control-ASO-treated mice, respectively, $n=6-10 /$ group, $P<0.002)$, and including insulin $(10 \mu \mathrm{U} / \mathrm{ml})$ in the perfusion buffer also had no significant effect on ketogenesis in either group $(0.07 \pm 0.02 \mu \mathrm{mole} / \mathrm{min} / \mathrm{g}$ vs. $0.42 \pm 0.12 \mu \mathrm{mole} /$ $\mathrm{min} / \mathrm{g}$ tissue in Hmgcs2- and control-ASO-treated mice, respectively, $n=4 /$ group, $P=0.03$; Figure $2 \mathrm{~A}$ ). Thus, Hmgcs2-ASO-treated mice are ketogenesis insufficient, irrespective of whether or not exogenous fat is delivered to the liver.

Proton NMR revealed a $65 \%$ increase of HGP in livers of fed-state ketogenesis-insufficient animals in the presence of $0.2 \mathrm{mM}$ sodium $\left[\mathrm{U}_{-}{ }^{13} \mathrm{C}\right]$ propionate $(1.01 \pm 0.12 \mu \mathrm{mole} / \mathrm{min} / \mathrm{g}$ vs. $0.61 \pm 0.09 \mu \mathrm{mole} /$ $\mathrm{min} / \mathrm{g}$ tissue in Hmgcs2- and control-ASO-treated mice, respectively, $n=12-13 /$ group, $P<0.02$; Figure $2 \mathrm{C})$, and excluding propionate had no significant effect on glucose production rate $(1.14 \pm 0.12 \mu \mathrm{mole} /$ $\mathrm{min} / \mathrm{g}$ vs. $0.73 \pm 0.09 \mu \mathrm{mole} / \mathrm{min} / \mathrm{g}$ tissue in Hmgcs2- and control-ASO-treated mice, respectively, $n=$ $6-10$ /group, $P<0.02$ ). As expected, $10 \mu \mathrm{U} / \mathrm{ml}$ insulin decreased $\mathrm{HGP}$ by more than $60 \%$ in both groups of animals (1.01 \pm 0.12 vs. $0.35 \pm 0.03, n=4-13$ /group, in insulin-free and insulin-containing perfusions, respectively, in Hmgcs2-ASO-treated animals, $P=0.01$; and $0.59 \pm 0.21$ vs. $0.25 \pm 0.06, n=4-12$ /group, $P$ $<0.01$, in insulin-free and insulin-containing perfusions, respectively, in control-ASO-treated mice; Figure 2C). In the absence of insulin, glycogenolytic flux was increased nearly 4-fold in livers of ketogenesis-insufficient animals $(0.58 \pm 0.1 \mu \mathrm{mole} / \mathrm{min} / \mathrm{g}$ vs. $0.15 \pm 0.03 \mu \mathrm{mole} / \mathrm{min} / \mathrm{g}$ in $\mathrm{Hmgcs} 2$ - and control-ASO-treated mice, respectively, $n=10-11$ /group, $P<0.001$; Figure 2D), an effect that was also independent of the presence of $\left[{ }^{13} \mathrm{C}\right]$ propionate in the perfusion buffer. Insulin decreased glycogenolytic flux by 5 -fold $(0.12 \pm 0.01$ $\mu \mathrm{mole} / \mathrm{min} / \mathrm{g}$ vs. $0.02 \pm 0.01 \mu \mathrm{mole} / \mathrm{min} / \mathrm{g}, n=4 /$ group, $P<0.001)$ in control-ASO-treated animals, and glycogenolytic flux was attenuated by insulin to a similar magnitude for Hmgcs2-ASO animals (Figure 2D). Fluxes of glycerol-derived HGP were modestly, but not significantly diminished by the inclusion of either $\left[{ }^{13} \mathrm{C}\right]$ propionate, insulin, or both, but were not different between Himgcs2- and control-ASO-treated mice (Figure 2E). HGP absolute flux rates from PEP also showed no $\left[{ }^{13} \mathrm{C}\right]$ propionate- or HMGCS2-dependent effect, but insulin modestly attenuated PEP flux in both groups (Figure 2F). Taken together, irrespective of the presence or absence of $\left[{ }^{13} \mathrm{C}\right]$ propionate, in the fed state, glycogenolysis dominated HGP in livers of ketogenesis-insufficient animals (58\% of HGP, versus $23 \%$ of HGP in controls, $P<0.001$; Figure 2G), whereas in livers of control animals, glycerol- and PEP-derived gluconeogenesis dominated HGP. Insulin attenuated glycogen's contribution to HGP in livers of both ketogenesis-insufficient and control animals, 
A

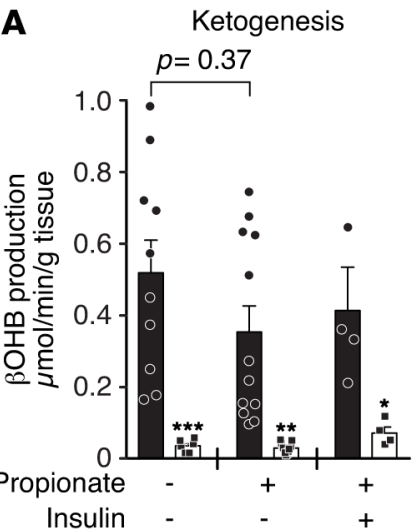

D

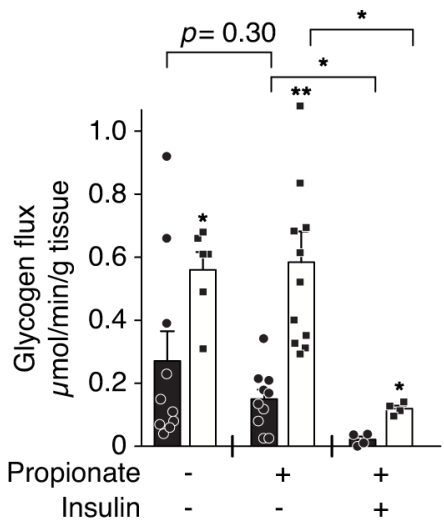

B

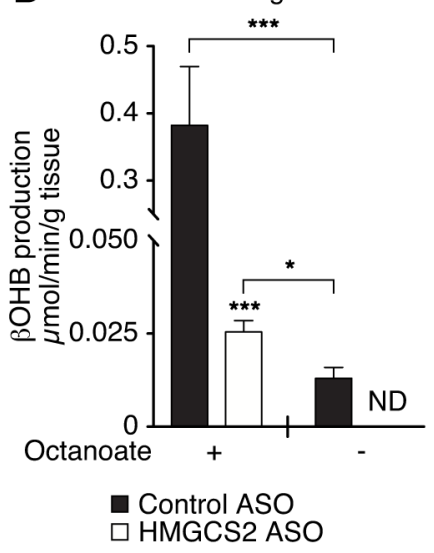

E

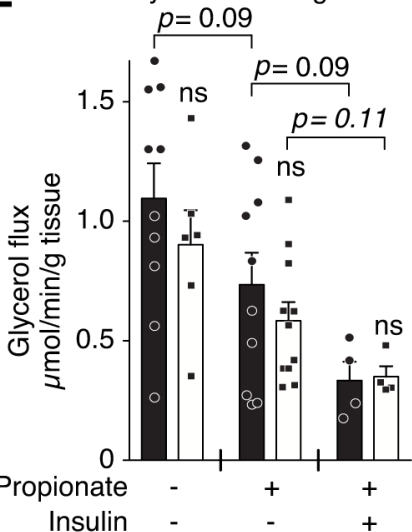

- PEP ם Glycerol $\square$ Glycogen

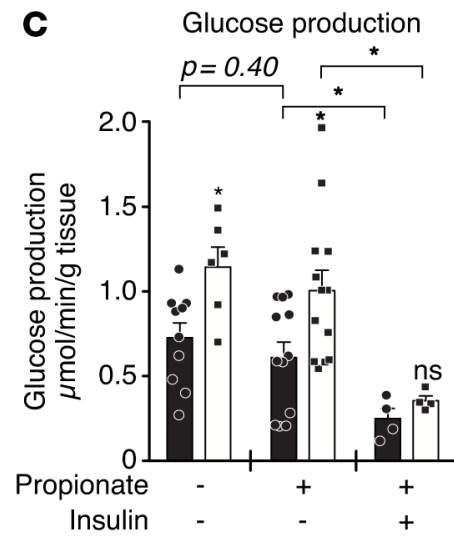

$\mathbf{F}$

PEP flux to glucose

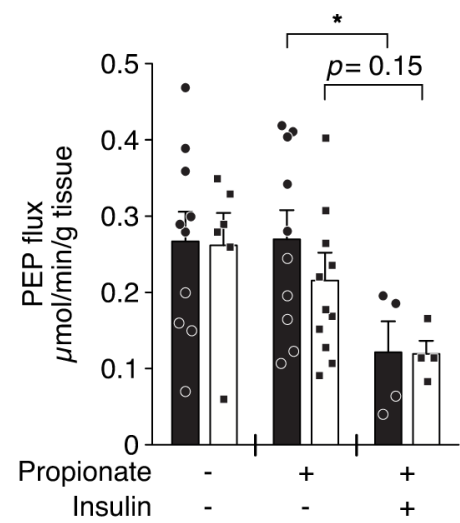

H Hepatic glycogen content

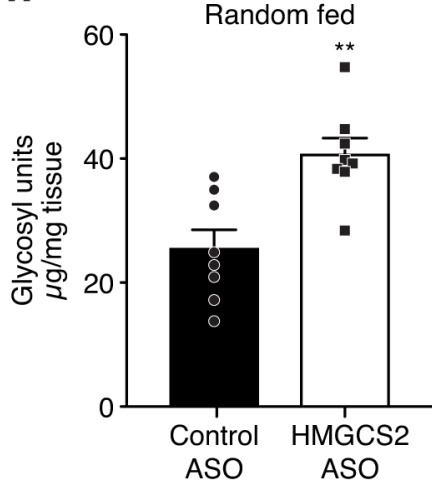

Figure 2. Ketogenesis-insufficient mice exhibit augmented glycogen-sourced hepatic glucose production. (A) $\beta O H B$ production quantified using hepatic venous effluent from 60-minute portal vein perfusions using oxygenated buffers containing $0.2 \mathrm{mM}$ unlabeled sodium octanoate and $3 \%{ }^{2} \mathrm{H}_{2} \mathrm{O}$, in the absence $(-)$ or presence $(+)$ of sodium [U-13 $\mathrm{C}$ propionate $(0.2 \mathrm{mM})$ or insulin $(10 \mu \mathrm{U} / \mathrm{ml})$. (B) $\beta$ OHB production in the absence $(-)$ or presence $(+)$ of sodium octanoate $(0.2 \mathrm{mM})$ from 60 -minute portal vein perfusions using oxygenated buffers. The first 2 bars on the left are presented for comparison and represent the same data as those presented in the middle 2 bars in panel A. (C) Glucose production, (D) glycogenolysis flux, (E) glycerol-supported flux to gluconeogenesis, and (F) phosphoenolpyruvate-supported (PEP-supported) flux to gluconeogenesis quantified as described for panel $\mathbf{A}$. (G) Relative contributions of glycogen, glycerol, and PEP to hepatic glucose production, derived from data presented in panels $\mathbf{C}-\mathbf{F}$. (H) Hepatic glycogen concentrations in random-fed (collected at $10 \mathrm{am}$ ) mice. $n=6$-13/group. ${ }^{*} P<0.05$; ${ }^{*} P<0.01$; ${ }^{* * *} P<0.001$; ${ }^{* * *} P<0.0001$. ns, not significant by 2-way ANOVA with Tukey's or Bonferroni's post hoc test, or Student's $t$ test as appropriate. Boxed values correspond to the effect of $10 \mu \mathrm{U} / \mathrm{ml}$ insulin in the presence of [ $\left.{ }^{13} \mathrm{C}\right]$ propionate, compared with the absence of insulin in the presence of $\left[{ }^{13} \mathrm{C}\right]$ propionate. ND, none detected.

but even in the presence of insulin, glycogenolysis sourced HGP to a much greater extent in liver of ketogenesis-insufficient animals than those of controls ( $34 \%$ vs. $10 \%$ in controls, $n=4 /$ group, $P<0.004)$.

Because ketogenic insufficiency was associated with increased glycogenolysis, we quantified hepatic glycogen pool sizes using livers from mice not undergoing portal vein perfusions. In livers harvested from age-, 
A

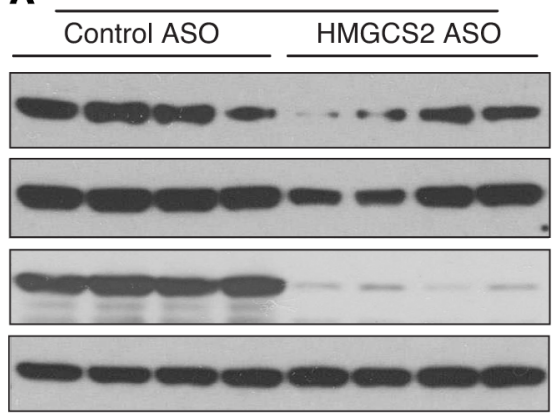

B

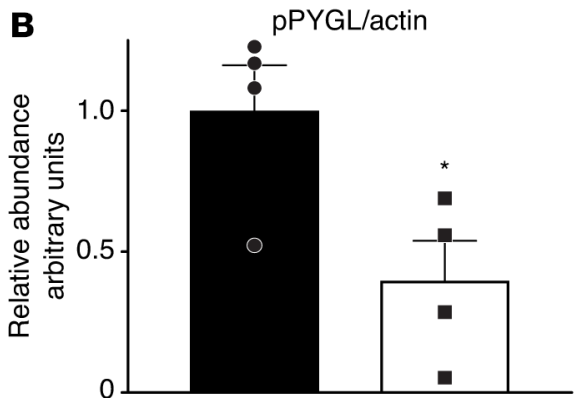

C
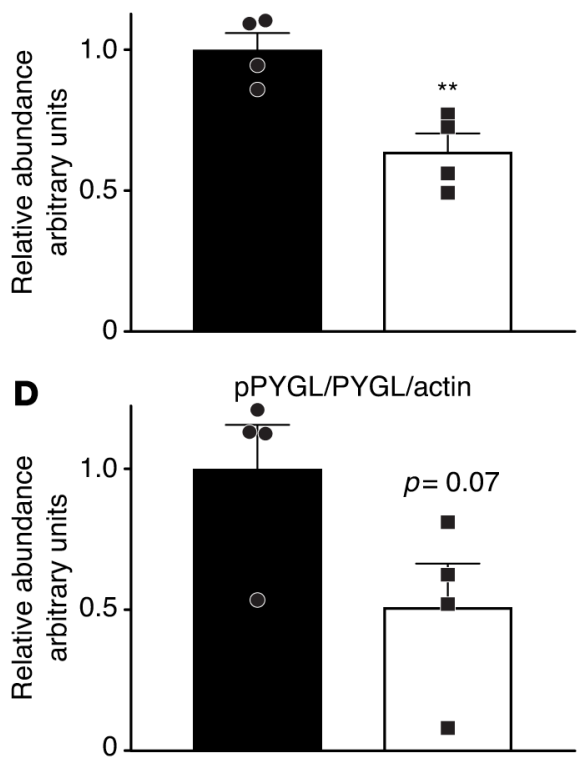

$\mathbf{E}$

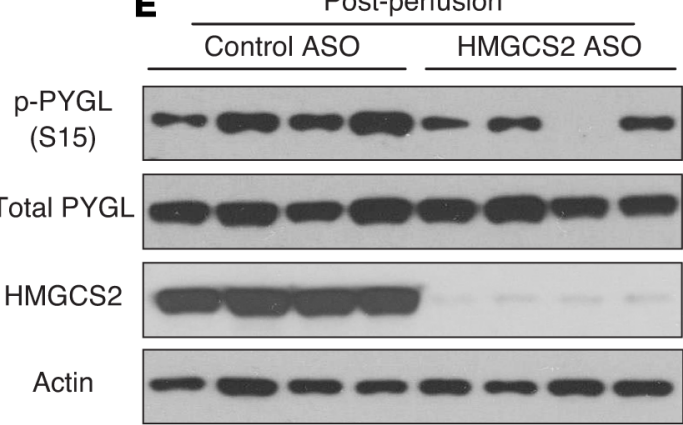

$\mathbf{F}$

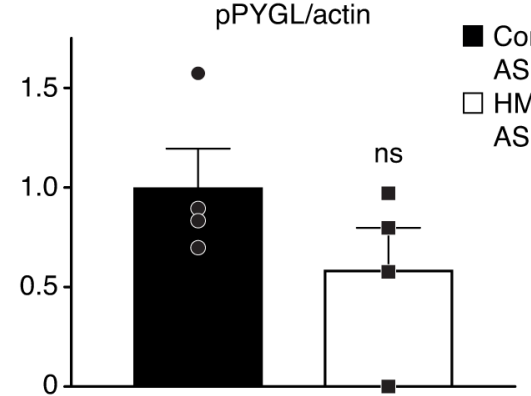

G 1 .

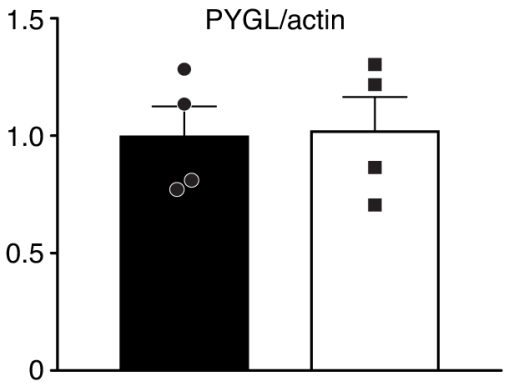

H

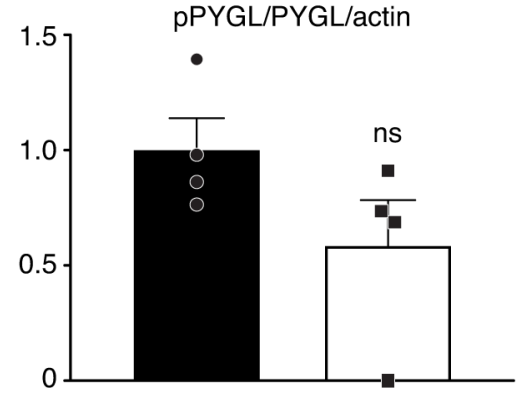

Figure 3. Diminished abundances of phosphorylated and total glycogen phosphorylase in livers of random-fed, but not postperfused ketogenesisinsufficient mice. Immunoblots for the phosphorylated (serine 15, activated) version of the liver isoform of glycogen phosphorylase ( $p-P Y C L)$, total PYGL, HMGCS2, and actin, acquired from protein lysates from livers of animals in the random-fed state (A-D) or livers acquired at the end of a 60-minute metabolic perfusion using octanoatecontaining oxygenated buffer (E-H). Densitometric quantifications of p-PYGL (B and $\mathbf{F}$ ), total PYGL ( $\mathbf{C}$ and $\mathbf{G})$, and the $\mathrm{p}$-PYGL/PYGL ratio ( $\mathbf{D}$ and $\mathbf{H})$, all normalized to actin. $n=4$ /group. ${ }^{*} P<$ $0.05 ;{ }^{* *} P<0.01$. ns, not significant by Student's $t$ test.

strain-, and sex-matched mice under random-fed conditions without restriction to food supply, the glycogen pool was surprisingly increased nearly $60 \%$ in livers of ketogenesis-insufficient mice $(40.7 \pm 2.6 \mu \mathrm{g} / \mathrm{mg}$ liver vs. $25.6 \pm 3.0 \mu \mathrm{g} / \mathrm{mg}$ liver in Hmgcs2- and control-ASO-treated mice, respectively, $n=8 /$ group, $P<0.01$; Figure $2 \mathrm{H}$ ). To determine if these collective observations could be linked to the function of the primary enzymatic mediators of glycogen turnover, glycogen phosphorylase and synthase, we used Western blots to quantify the abundances of both total and phosphorylated fractions of these enzymes in livers of animals in the random-fed and postperfusion states, because hormonally regulated phosphorylation of these enzymes activate or inhibit their specific activities, respectively (3). In the random-fed state, the abundance of the liver-specific isoform of glycogen phosphorylase in its phosphorylated (active) state (p-PYGL) was significantly diminished more than $50 \%$ ( $n=4$ /group, $P=0.03$; Figure 3, A and B) in the livers of ketogenesis-insufficient animals. Most of this decrease appeared to be attributable to an approximately $40 \%$ decrease in the abundance of the total PYGL pool ( $n=4$ /group, $P=0.007$; Figure 3 , A and C), such that the diminution of p-PYGL, normalized to the total PYGL pool, did not meet statistical significance (Figure 3D). After perfusion, there were no 
A

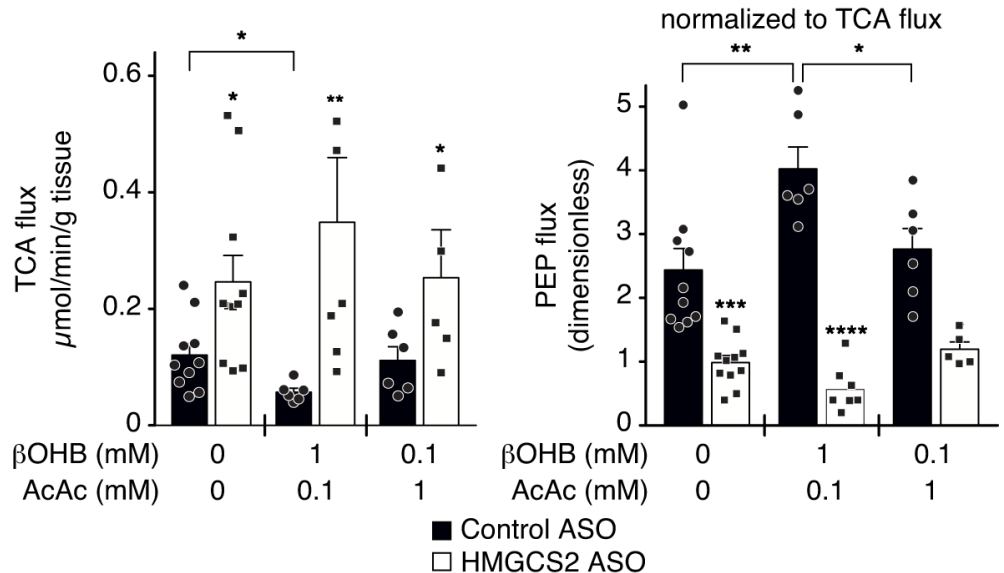

C

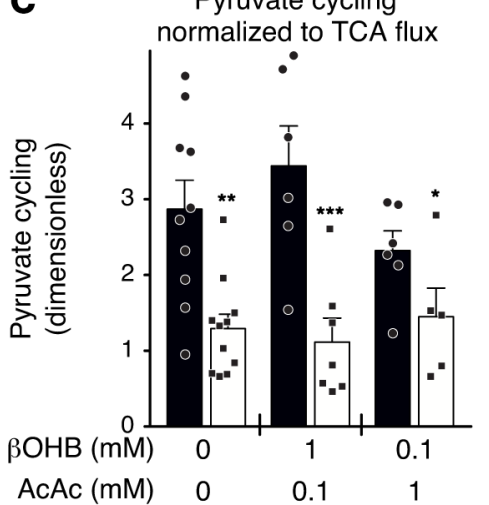

B

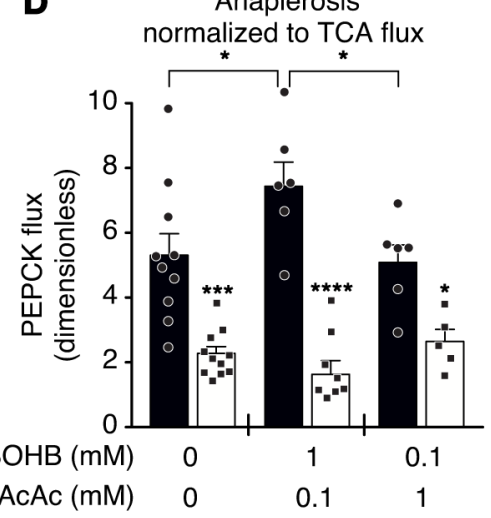

Figure 4. Increased TCA cycle flux is unmatched by PEP flux, pyruvate cycling, and anaplerosis in livers of ketogenesisinsufficient animals. (A) TCA cycle flux; and (B) PEP flux, (C) pyruvate cycling flux, and (D) anaplerotic flux, all normalized to TCA cycle flux, quantified by ${ }^{2} \mathrm{H}$ - and ${ }^{13} \mathrm{C}-\mathrm{NMR}$ of monoacetone $\left[{ }^{3} \mathrm{C} /{ }^{2} \mathrm{H}\right]$ glucose derivatized from hepatic venous effluent ${ }^{13} \mathrm{C} /{ }^{2} \mathrm{H}$-labeled glucose from 60 -minute perfusions using oxygenated buffer that included $0.2 \mathrm{mM}$ unlabeled sodium octanoate, $3 \%{ }^{2} \mathrm{H}_{2} \mathrm{O}$, and $0.2 \mathrm{mM}$ sodium $\left[\mathrm{U}-{ }^{13} \mathrm{C}\right.$ ]propionate, in the absence or presence of supplemented unlabeled ketone bodies at the indicated concentrations. $n=6-13$ /group. ${ }^{*} P$ $<0.05$; ${ }^{* *} P<0.01$; ${ }^{* * *} P<0.001$; ${ }^{* * *} P<0.0001$ by 2-way ANOVA with Tukey's post hoc test.

significant differences in p-PYGL or PYGL, and the total PYGL pool size became equalized (Figure 3, E-H). Finally, there were no differences in the phosphorylated fractions or total pool sizes of glycogen synthase (GS) in the random-fed or postperfusion states (Supplemental Figure 4). Taken together, these quantifications of glycogenolysis flux, hepatic glycogen pool sizes, and enzyme abundance all suggest that livers of ketogenesisinsufficient mice are poised to store hepatic glycogen, likely due to diminished PYGL activity, but that in perfused livers, glycogenolysis from these larger pools becomes unrestrained. These collective observations are also consistent with the very mild hyperglycemia and normal insulinemia that we previously observed in these animals in vivo, contrasted here with substantially increased hepatic glucose output due to glycogenolysis in the ketogenesis-insufficient perfused liver (19).

Ketogenic insufficiency increases TCA cycle flux, unmatched by gluconeogenesis, pyruvate cycling, and anaplerosis. Given the intimate metabolic connection between hepatic ketogenesis and TCA cycle flux, and in turn the tight coupling among TCA cycle flux, hepatic anaplerosis/cataplerosis, and PEP-derived gluconeogenesis, ${ }^{13} \mathrm{C}$-NMR signal magnitudes were obtained from monoacetone $\left[{ }^{13} \mathrm{C} /{ }^{2} \mathrm{H}\right]$ glucose derived from hepatic venous effluent ${ }^{13} \mathrm{C} /{ }^{2} \mathrm{H}$-labeled glucose from portal vein perfusions, allowing quantification of hepatic oxidative fluxes. Livers of ketogenesis-insufficient mice exhibited a 2 -fold increase in TCA cycle flux in the fed state $(0.25 \pm 0.05 \mu \mathrm{mole} / \mathrm{min} / \mathrm{g}$ vs. $0.12 \pm 0.02 \mu \mathrm{mole} / \mathrm{min} / \mathrm{g}$ in Hmgcs 2 - and control-ASO-treated mice, respectively, $n=10-11 /$ group, $P<0.03$; Figure $4 \mathrm{~A}$ ). To confirm that this marked increase could not be attributed to the relative absence of ketone bodies themselves, portal vein perfusions were also performed using buffers that also contained freshly prepared either $1 \mathrm{mM} \beta O H B / 0.1 \mathrm{mM}$ AcAc, or separately, $1 \mathrm{mM}$ AcAc/0.1 mM $\beta O H B$. In either mitochondrial redox potential condition, TCA cycle flux remained increased in livers of ketogenesis-insufficient animals (Figure 4A), indicating that ketogenic flux impairment, rather than ketone bodies themselves, was responsible for the increase in TCA flux. Consistent with expectations given the effect of reduced redox potential, TCA flux was modestly (but significantly with respect to its original value) diminished in the $1 \mathrm{mM} \beta \mathrm{OHB} / 0.1 \mathrm{mM}$ AcAc group, and only in control animals.

Previous observations have indicated that, in the setting of obesity (compared to lean controls), increased hepatic TCA flux is matched by commensurate rises in gluconeogenesis (PEP flux), pyruvate 
A

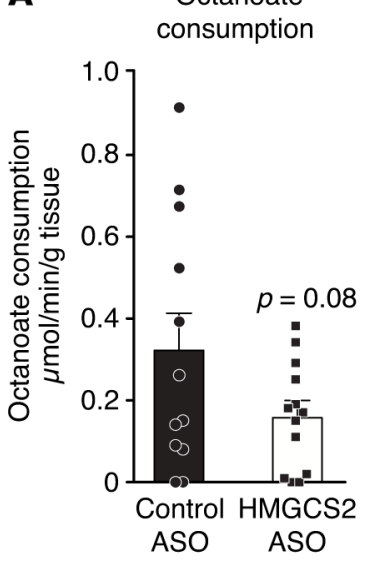

B

Acetyl-CoA
balance

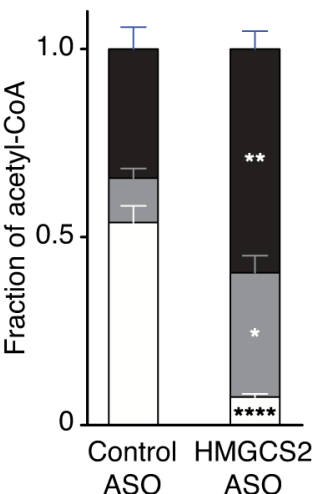

Unaccounted

C Mitochondria
Acetyl-CoA

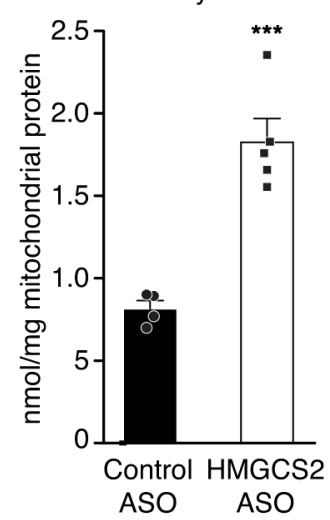

TCA Flux $\square$ Ketogenesis

D

Total hepatic Acetyl-CoA content

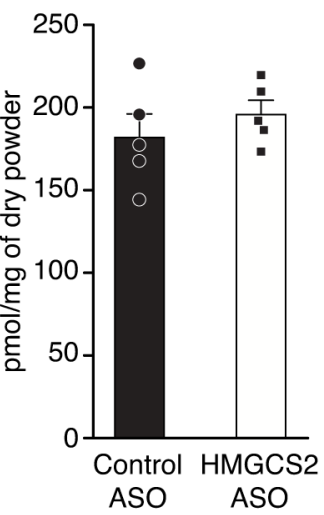

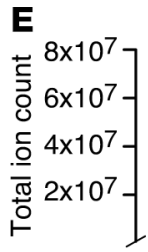

3-oxo-octanoic acid

$$
\mathbf{F}
$$

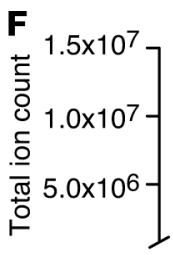

3-oxo-decanoic acid
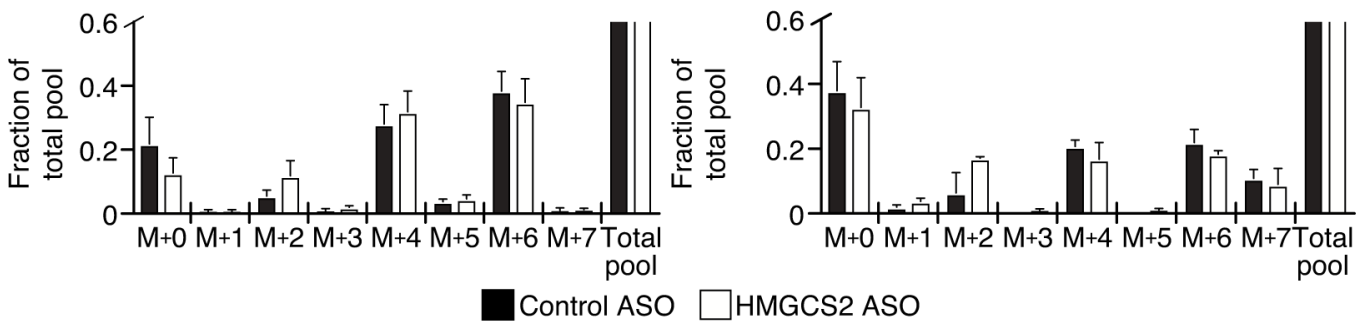

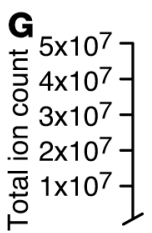

Butyryl-CoA

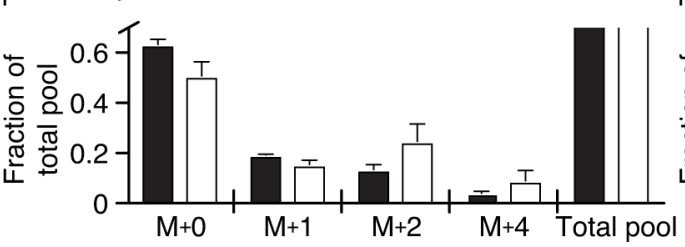

H $6 \times 10^{7} \quad$ Hydroxybutyryl-CoA

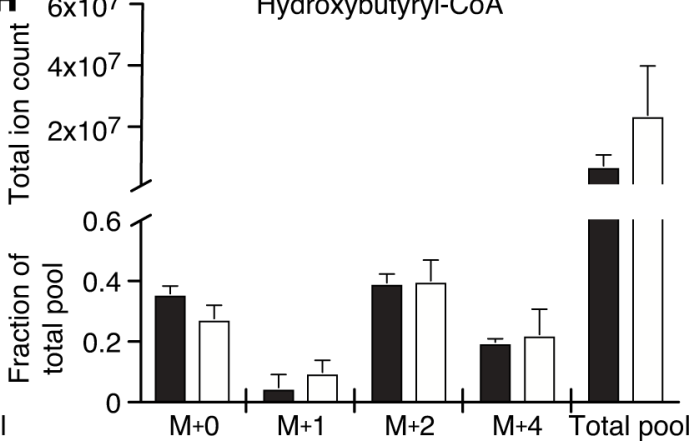

Figure 5. Acetyl-CoA fates in ketogenic insufficiency. (A) Octanoate consumption rates and (B) derived acetyl-CoA fates of livers of animals perfused with $0.2 \mathrm{mM}$ unlabeled octanoate-containing oxygenated perfusion buffer from the experiments whose data are presented in Figures 2-4. Note that little octanoate is esterified to glycerol, and much of the unaccounted fraction in controls is attributable to AcAc, while very little of the unaccounted fraction can be sourced to AcAc in ketogenic insufficiency. (C) LC-MS/MS measurements of acetyl-CoA from extracts of isolated mitochondria, normalized to mitochondrial protein content, or (D) total liver tissue, normalized to tissue mass, from livers harvested from unperfused mice. (E-H) Untargeted LC/MS metabolomics from liver extracts derived from portal vein perfusions using oxygenated buffer containing $0.2 \mathrm{mM}$ sodium $\left[1,2,3,4-{ }^{13} C_{4}\right]$ octanoate. Collections were acquired in the negative-ion mode after chromatographic separation. Isotopologues reflect ${ }^{13} \mathrm{C}$ incorporation in 2-atom units consistent with products from labeled acetyl-CoA export from mitochondria. (E) 3-Oxo-octanoic acid, whose monoisotopic mass is 158.0943, putatively assigned to $\mathrm{m} / \mathrm{z} 217.1081\left[\mathrm{M}+\mathrm{CH}_{3} \mathrm{COO}\right]^{-}$(acetylated molecular ions are common under the HILIC mobile phase conditions employed); $M+0, M+2, M+4$, and $M+6$ isotopologues are equally abundant in both genotypes, suggestive that ketogenic insufficiency does not alter this metabolite's labeling. (F) 3-Oxo-decanoic acid, whose monoisotopic mass is 186.1256 , putatively assigned to $\mathrm{m} / \mathrm{z} 245.1393\left[\mathrm{M}+\mathrm{CH}_{3} \mathrm{COO}\right]$; $\mathrm{M}+0$, $M+2, M+4, M+6$, and $M+8$ isotopologues are equally abundant in both genotypes. (G) Butyryl-CoA, whose monoisotopic mass is 837.1571 , putatively assigned to $\mathrm{m} / \mathrm{z} 417.5715[\mathrm{M}-2 \mathrm{H}]^{2-} ; \mathrm{M}+0$ and $\mathrm{M}+2$ isotopologues are equally abundant in both genotypes (parent monoisotopic masses that acquire $2 \mathrm{e}^{-}$in ionization result in a $\mathrm{m} / \mathrm{z}$ that is half of the expected monoisotopic mass for the molecular ion). (H) Hydroxybutyryl-CoA, whose monoisotopic mass is 853.1520 , putatively assigned to $m / z 425.5691[M-2 H]^{2-} ; M+0, M+2$, and $M+4$, isotopologues are equally abundant in both genotypes. $n=$ 4-6/group. ${ }^{* *} P<0.001 ;{ }^{* * *} P<0.0001$ by Student's $t$ test. 
cycling, and anaplerosis, such that the ratios of each of these fluxes, normalized to TCA flux, remain relatively constant (10). In our experiments in long-term wild-type HFD-fed animals, these fluxes were elevated when normalized to TCA cycle, likely indicating that reducing equivalents from $\beta$-oxidation/ketogenesis drive increased energy charge (Figure 1 and Supplemental Figure 3F). Intriguingly, in livers of chow dietfed ketogenesis-insufficient animals in the fed state, the increase in TCA flux was not matched by increases in PEP flux, pyruvate cycling, or anaplerosis, such that each of these fluxes becomes significantly diminished relative to TCA flux (Figure 4, B-D). As observed with regard to TCA flux rates, supplementation of ketone bodies did not significantly alter the effects of ketogenic insufficiency on PEP flux, pyruvate cycling, or anaplerosis. Moreover, supplemented ketone bodies did not abrogate the augmentation of HGP and glycogenolysis in livers of ketogenesis-insufficient animals, and overall glucose sourcing was not significantly altered (Supplemental Figure 5). Taken together, these observations suggest that the relationships between anaplerotic/cataplerotic flux and TCA cycle flux vary among metabolic contexts, underscoring the need to consider all contributors to hepatic energy charge, particularly in pathological conditions that include NAFLD progression to NASH.

Ketogenic insufficiency redistributes mitochondrial acetyl-CoA fates. Increased TCA cycle flux in livers of ketogenesis-insufficient animals, even in the fed state, is not unexpected; as long as oxaloacetate pools are sufficient, $\beta$-oxidation-derived acetyl-CoA, whose pools are tightly regulated in mitochondrial matrix, can be diverted to citrate synthase. We hypothesized that citrate synthase flux alone would not account for all of the acetyl-CoA that fails to be incorporated into ketone bodies, even in the fed state. The scope of unaccounted acetyl-CoA was first determined by using ${ }^{1} \mathrm{H}-\mathrm{NMR}$ to quantify octanoate extracted from perfused livers, which revealed a $50 \%$ decrease in perfused livers of ketogenesis-insufficient animals that did not quite meet statistical significance (Figure 5A). As expected, allocation of acetyl-CoA to the TCA cycle was relatively increased (from $12 \%$ to $33 \%$ ), and to ketogenesis was markedly decreased (from $54 \%$ to $7 \%$ ) in livers of ketogenesis-insufficient animals (Figure 5B). Thus, the balance of potentially unaccounted acetylCoA was 74\% larger (increased from 34\% in controls, to 59\%) in livers of ketogenesis-insufficient animals, and furthermore, much of the unaccounted fraction in controls is attributable to AcAc, while little of the $59 \%$ unaccounted balance in ketogenic insufficiency will be attributable to AcAc.

To determine the additional fates of acetyl-CoA, we undertook a multiscale approach. First, we used targeted LC-MS/MS to determine that steady-state acetyl-CoA concentrations were increased 2-fold in mitochondria isolated from unperfused harvested livers of ketogenesis-insufficient animals, but were normal in whole liver tissue (Figure 5, C and D). Second, we applied an isotope-tracking untargeted metabolomics approach to map, across the metabolome, the fates of $0.2 \mathrm{mM}$ sodium $\left[1,2,3,4-{ }^{13} \mathrm{C}_{4}\right]$ octanoate that was delivered via portal vein perfusions $(19,25) . \mathrm{m} / \mathrm{z}$ ratios corresponding to putative identifications for unlabeled and incrementally $\left[{ }^{13} \mathrm{C}\right]$ octanoate-derived isotopologue intermediates of cytoplasmic lipid biosynthesis (3-oxo-octanoic acid, 3-oxo-decanoic acid, butyryl-CoA, and hydroxybutyryl-CoA) were observed equally in liver tissue of control and ketogenesis-insufficient animals (Figure 5, E-I). The observed isotopologue patterns for each of these 4 intermediates derived from $\beta$-oxidation of $\left[1,2,3,4-{ }^{13} \mathrm{C}_{4}\right]$ octanoate can only arise from futile recycling of $\left[1,2-{ }^{13} \mathrm{C}_{2}\right]$ acetyl-CoA into cytoplasmic lipid synthesis, rather than as intermediates of $\beta$-oxidation alone or due to direct incorporation of $\left[1,2,3,4-{ }^{13} \mathrm{C}_{4}\right]$ octanoate into cytoplasmic enzymatic acyl chain-lengthening pathways (26). As there were no differences observed in the labeled abundances of these fatty acid synthetic intermediates in livers of ketogenesis-insufficient animals, hepatic ketone bodies in the fed state may not primarily furnish recycled acetyl-CoA to cytoplasmic lipid synthesis, or if they do, alternative mechanisms for acetyl-CoA mitochondrial efflux into cytoplasmic lipid synthetic pathways (i.e., citrate or acetylcarnitine efflux) compensate for ketogenic insufficiency. Finally, the absence of any difference in labeling of hydroxybutyryl-CoA (e.g, $\beta \mathrm{OHB}-\mathrm{CoA}$; Figure $5 \mathrm{H}$ ) in livers of ketogenesisinsufficient mice suggests that hepatic $\beta \mathrm{OHB}-\mathrm{CoA}$, an intermediate of lysine catabolism and $\beta$-oxidation itself, is a poor reporter of hepatic ketogenesis.

Ketogenic insufficiency and fatty liver states promote marked accumulation of a canonical late endosomal lipid class. To determine how ketogenic insufficiency reprograms the metabolome of the mouse liver in the fed state we performed multiscale analysis through MS-based profiling. First, untargeted high-resolution LC/MS metabolomics was performed in livers directly harvested (without perfusion) from control and ketogenesis-insufficient mice, detecting 7,949 chemical features, of which 758 features showed more than a 2-fold magnitude change with associated $P$ value less than 0.05 (Supplemental Figure 6A and Supplemental Table 1). Putative identification could be assigned to 105 of the features using the METLIN 
A Dysregulated features in ketogenesis insufficient liver

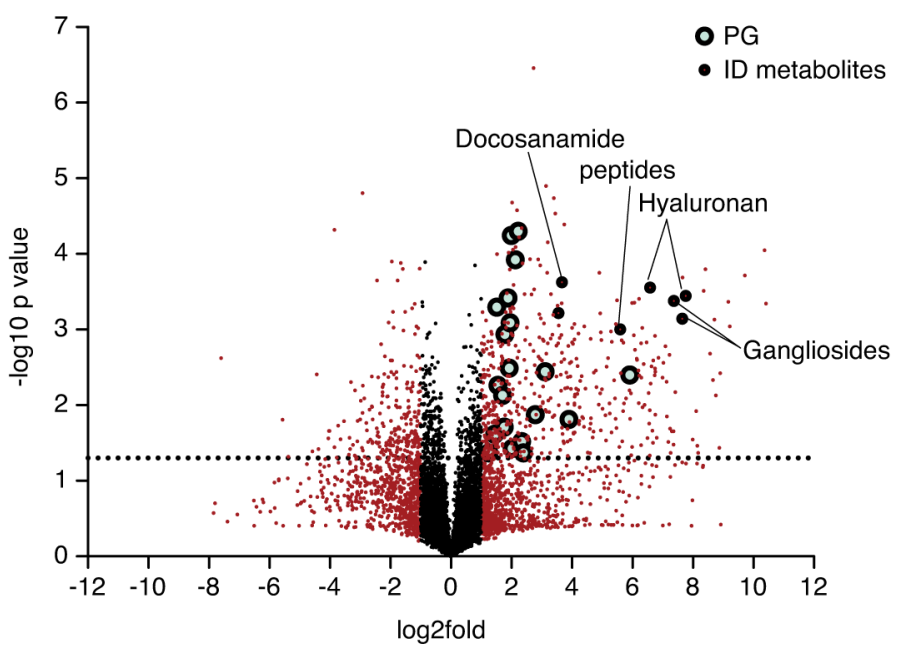

$c$ PA, PG, and CL hepatic concentrations

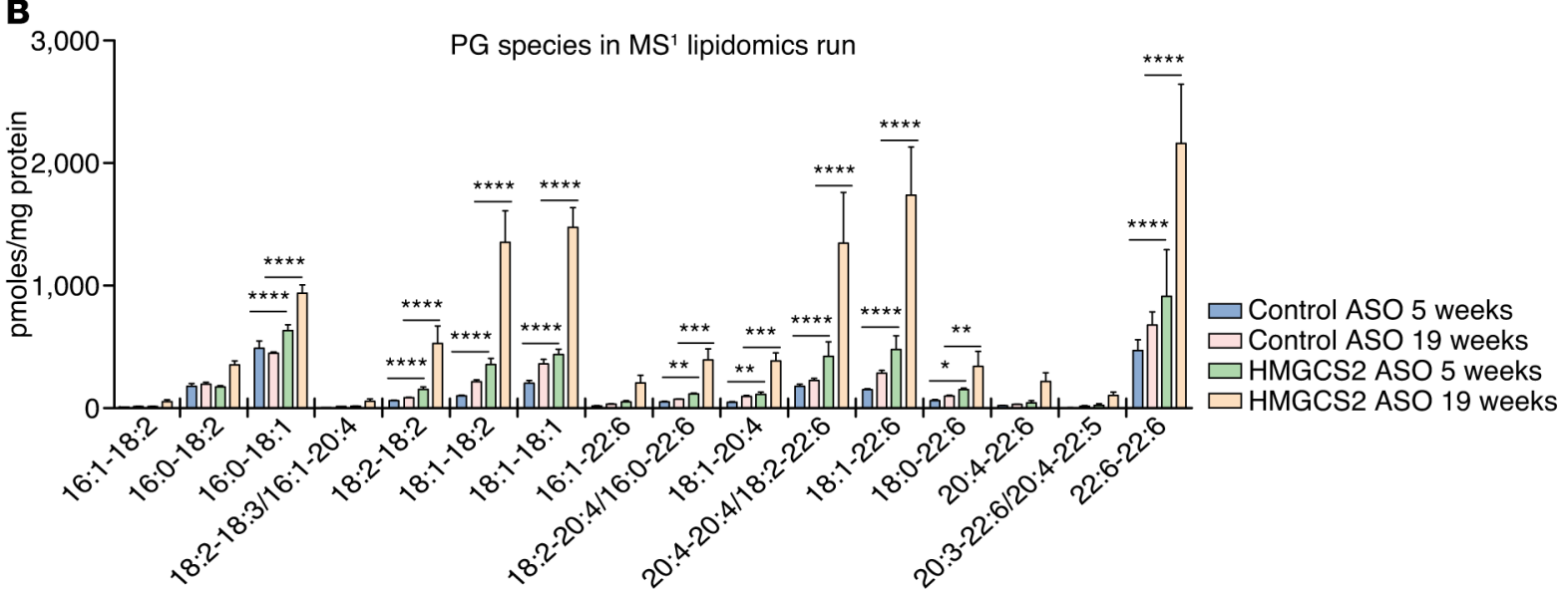

Figure 6. Accumulation of numerous putative phosphatidylglycerol (PG) species in ketogenesis-insufficient livers. (A) Volcano plot of analyzed untargeted metabolomics signals from snap-frozen (unperfused) livers revealed, among numerous up- and downregulated chemical features, 19 upregulated $\mathrm{m} / \mathrm{z}$ ratios mapping to putative $\mathrm{PG}$ species. (B) High-resolution shotgun lipidomics (MS') confirmed progressive accumulation of numerous putative PG species in livers of snap-frozen ketogenesis-insufficient livers. (C) Radial plot of molar concentrations of the total pools of putative PC species, their precursors, phosphatidic acids (PAs), and their products, cardiolipins (CLs). Each segment radiating from the center represents an individual biological replicate. $n=4$-5/group. ${ }^{*} P<0.05 ;{ }^{* *} P<0.01$; ${ }^{* * *} P<0.001$; ${ }^{* * *} P<0.0001$ by 2 -way ANOVA with Bonferroni's post hoc test.

database (Supplemental Table 2). Nearly $70 \%$ of the assigned and significantly upregulated metabolites were putative hyaluronan, decanamide, peptides, and gangliosides (Figure 6A). Intriguingly, 19 of the noted putative identifications mapped to upregulated phosphatidylglycerol (PG) lipid species in livers of ketogenesis-insufficient animals (blue-filled circles in Figure 6A). Therefore, targeted multidimensional MS-based shotgun lipidomics (MDMS-SL) was undertaken to formally confirm and quantify numerous classes of lipid species. Notably, livers of ketogenesis-insufficient animals failed to show class-wide differences, compared with livers of control animals, among TAGs, DAGs, lysophosphatidylethanolamines (LPEs), phosphatidylinositols (PIs), lysophosphatidylcholines (LPCs), phosphatidylserines (PSs), phosphatidylethanolamines (PEs), ceramides, or sphingomyelins (SMs) (Supplemental Figure 6, B and C). However, consistent with the putative identities established by untargeted LC/MS, numerous PG species were augmented in livers of ketogenesis-insufficient animals, which accumulated with increasing duration of ketogenic insufficiency (Figure 6B). Surprisingly, abundances of PG precursors phosphatidic acid (PA) species, and PG biosynthetic products, cardiolipin species (CL), were unaffected (Supplemental Figure 7A and Figure 6C). Similarly, abundances of mRNAs encoding mediators of PG synthesis and catabolism were unchanged between control and ketogenesis-insufficient livers (Supplemental Figure 7, A and B). These discrepant observations shifted our attention to a class of PG structural isomers that are usually 

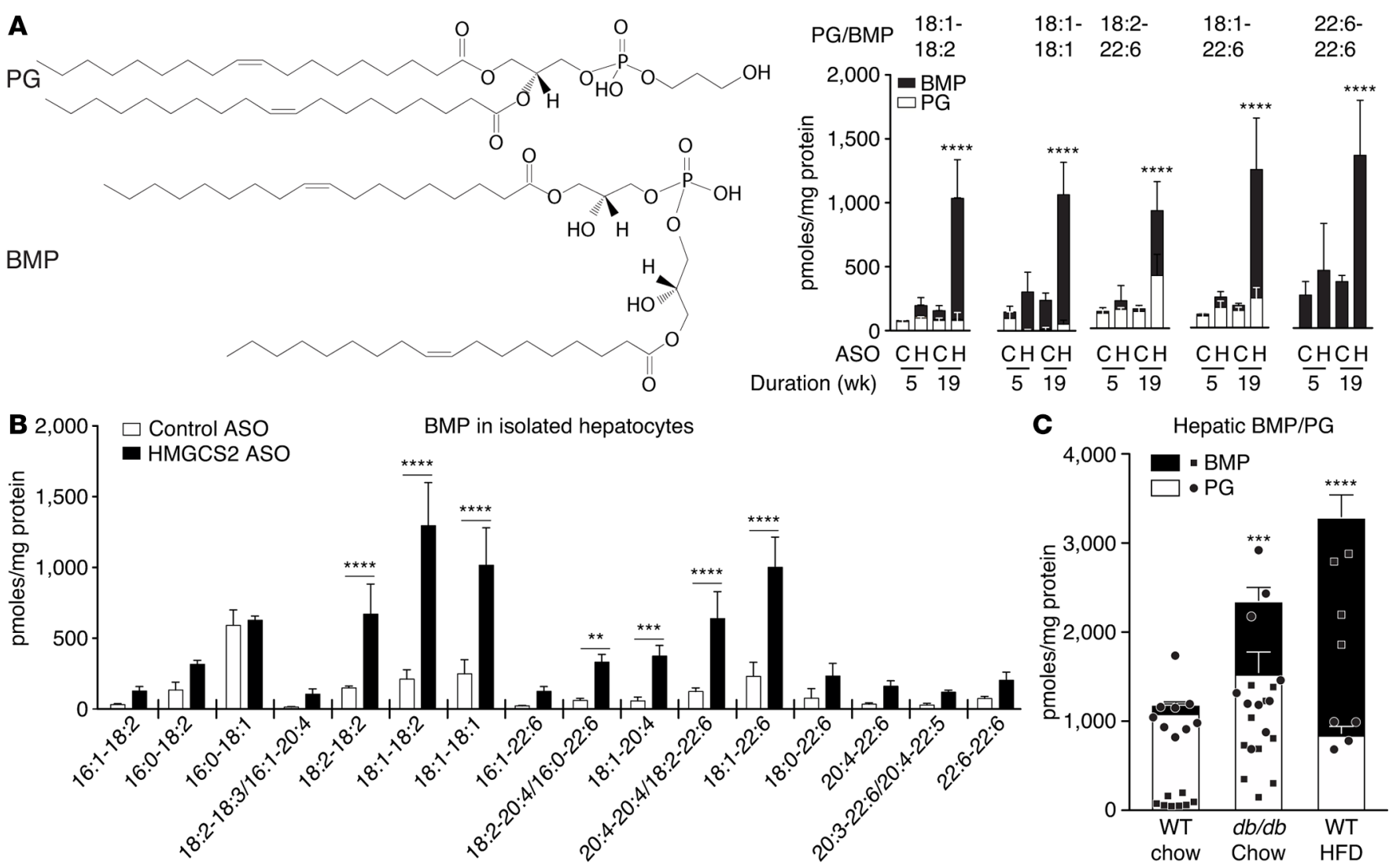

Figure 7. MS/MS analysis allows assignment of putative PGs as bis(monoacylglycero)phosphate (BMP) species in multiple models of disordered hepatic metabolism, confirmed by tandem MS. (A) Structural isomers phosphatidylglycerol (PG) and bis(monoacylglycero)phosphate (BMP, left); molar concentrations of 4 sets of PG/BMP, fully confirmed and speciated using MS/MS (right), revealing progressive accumulation, particularly of BMPs, in livers of ketogenesis-insufficient animals. (B) MS confirmation of BMP species abundances in primary cultured hepatocytes. (C) Total PG/BMP pool concentrations in livers of wild-type control mice, $d b / d b$ mice, and wild-type mice maintained on high-fat diet for 8 weeks. C, control ASO; H, Hmgcs2 ASO. $n=4-9 / g r o u p$. ${ }^{* *} P<0.01 ;{ }^{* *} P<0.001 ;{ }^{* * *} P<0.0001$ by Student's $t$ test or 1-way ANOVA with Tukey's post hoc test, as appropriate.

measured in low abundance, except within late endosomes and lysosomes, the bis(monoacylglycero)phosphates (BMPs, also known as lysobisphosphatidic acids) (Figure 7A) $(27,28)$. Indeed, tandem mass spectrometric analysis confirmed that livers of ketogenesis-insufficient animals accumulated predominantly BMP lipid species, rather than PGs (Figure 7B). To assess whether BMPs are accumulating in hepatocytes, and not in other neighboring cell types in the liver, we isolated hepatocytes from livers of ketogenesis-insufficient and control animals, confirming that the majority of BMP species were increased in hepatocytes (Figure 7C). Interestingly, abundance of the ABHD6 enzyme, capable of catabolizing BMPs in late endosomes, was unchanged between control and ketogenesis-insufficient livers (Supplemental Figure 7C) $(29,30)$. While there is relatively little literature regarding BMPs, the most prevalent biological function reported is the modulation of cholesterol transport through late endosomes and lysosomes $(27,28)$. Total hepatic cholesterol contents were normal in livers of ketogenesis-insufficient animals, suggesting that (a) BMPs may also play alternate biological functions, and (b) perturbation of mitochondrial acetylCoA homeostasis via HMGCS2 manipulation may not have a major impact on HMGCS1-dependent metabolism (Supplemental Figure 7D). Nonetheless, association of the BMP lipid class with NAFLD progression may be a generalized biomarker, as livers of $d b / d b$ mice, and HFD-fed wild-type mice, also exhibit increased abundance of the BMP lipid class, despite normal ketonemia (Figure 7C and Supplemental Figure 7, E-H). Taken together, the results show that both ketogenesis-insufficient livers and other models of NAFLD exhibit progressive accumulation of BMPs, a canonical late endosomal lipid class. These observations confirm what we believe to be a novel function of ketogenesis as a mitochondrial pathway that integrates metabolic and signaling pathways among cellular compartments in the fed state, thereby coordinating key aspects of hepatic physiological homeostasis. 


\section{Discussion}

Ketogenesis offers an energetically favorable route for clearance of fat whose abundance supersedes the oxidative disposal capacity of the hepatic TCA cycle (6). Because ketogenesis is activated in carbohydrate restriction and other physiological states in which hepatic glycogen is depleted, this pathway is not commonly considered to serve an adaptive role in the carbohydrate-fed state. The findings herein implicate a significant role for hepatic ketogenesis in regulating complex metabolic pathways in the liver in a classically non-ketogenic, hepatic glycogen-replete state. In the fed state, ketogenic insufficiency results in 2 key changes in central hepatic metabolic fluxes: (a) doubling of TCA cycle flux, unaccompanied by commensurate increases in gluconeogenesis, pyruvate cycling, or anaplerosis and (b) predisposition to increased hepatic glucose production, largely attributable to a marked increase in glycogenolysis. As the liver constitutes only $4 \%$ of body weight and only $10 \%$ of whole-body energy expenditure, doubling hepatic TCA flux does not appear to cause an obvious increase in organismal energy expenditure, as ketogenesis-insufficient animals maintained on normal chow exhibit normal caloric intake and body weights $(19,31)$. In fact, it is likely that the octanoate-stimulated increase in hepatic TCA cycle flux represents a metabolic compensation for lost $\beta$-oxidation/ketogenesis that ultimately maintains equivalent mitochondrial energy potential to that of control livers.

The primary purposes of ketogenesis are to (a) support energy harvest from $\beta$-oxidation when fatty acid supply exceeds energetic demand from the higher-payoff TCA cycle, (b) reoxidize a portion of the mitochondrial $\mathrm{NADH}$ pool through the $\beta \mathrm{OHB}$ dehydrogenase reaction, and (c) after secretion, provide an energy source for non-hepatocytes, useful in states of carbohydrate restriction. Thus, primary effects of ketogenesis coordinate hepatocyte metabolic function, but this impact, and its potential ability to dynamically coordinate liver metabolism, is not commonly considered. Livers of octanoate-fueled ketogenesisinsufficient mice exhibited augmented TCA flux and elevated mitochondrial acetyl-CoA concentration, which together would normally be expected to yield a commensurate rise in gluconeogenesis and anaplerosis. ATP is a substrate, and acetyl-CoA is an allosteric activator of anaplerotic pyruvate carboxylase, while endergonic gluconeogenic flux requires 6 moles of nucleotide triphosphate plus 2 moles of NADH per mole of glucose produced. Instead, gluconeogenic and anaplerotic fluxes were normal in ketogenesisinsufficient livers, suggesting that energy charge in ketogenesis-insufficient livers is normal. As a primary energy consumer, gluconeogenesis is a surrogate for overall hepatic energy charge, even with significant redistributions of energy economy, such as ketogenic insufficiency, in which over half of the energy production conduit is lost with ketogenic ablation in the octanoate-fueled liver. Importantly, despite concern for confounding and undesired metabolic effects of using $\left[{ }^{13} \mathrm{C}\right]$ propionate as a tracer (32), our observations are concordant with observations from numerous groups supporting the absence of effects of tracer concentrations of propionate on HGP, or its contributing component fluxes (10, 24, 33, 34). Moreover, our use of this method also confirms prior conclusions using similar methods in rodent and human studies that fatty liver is in a high energy turnover state $(10,13)$; specifically, we observed in wild-type mice fed an HFD elevations in gluconeogenesis (energy consumer) and ketogenesis (energy producer), despite only modest increases in TCA cycle flux. Thus, it may be argued that augmenting ketogenesis to unload excess fat could exacerbate the energy surplus of the fatty liver, and further drive deleterious HGP and oxidative stress. The counter-argument to this perspective is that ketogenesis generates fewer reducing equivalents than does the TCA cycle (from long-chain fatty acids, $\sim 6$ reducing equivalents, compared with $\sim 3$ from ketogenesis; see ref. 10), and as a higher-capacity conduit, may be an effective mechanism to distribute excess reducing equivalents to extrahepatic tissues in a form that can be readily oxidized. Indeed, clinical success of sodium-glucose cotransporter-2 (SGLT2) inhibition, an oral hypoglycemic therapy that increases both ketogenesis and HGP, suggests that in select metabolic contexts, ketogenic augmentation may be advantageous (35-37).

In addition to increased TCA cycle flux, the ketogenesis-insufficient liver enlists multiple mechanisms to maintain normal energy charge, including (a) diminished extraction of the available fat, (b) maintenance of modestly higher steady-state acetyl-CoA concentrations in mitochondria, and (c) disposal of over half of the energy stored in fatty acids to destinations not identified by the stable-isotope-tracking NMR and high-resolution untargeted metabolomics platforms used herein. Our previous observations indicated medium- and short-chain acylcarnitines (quantified using targeted approaches, but unmeasured through this untargeted metabolomics platform) are likely conduits for $\beta$-oxidation-derived intermediates not terminally disposed in the TCA cycle or diverted to ketogenesis (19). Interestingly, high-resolution isotope-tracking untargeted 
metabolomics studies of livers perfused with $\left[1,2,3,4-{ }^{13} \mathrm{C}_{4}\right]$ octanoate did confirm recycling of mitochondrial $\beta$-oxidation-derived acetyl-CoA into cytoplasmic lipogenesis, likely mediated through citrate efflux, but this was not increased in ketogenesis-insufficient livers. However, ketogenesis-insufficient livers perfused from labeled carbohydrate substrates (lactate/pyruvate) previously revealed numerous substantially upregulated intermediates linked to de novo lipogenesis metabolites (19). These observations indicate that carbohydrate but not octanoate-derived acetyl-CoA is preferentially sourced to lipid in ketogenesis-insufficient livers.

The perfused liver model employed herein confers both experimental limitations and important insights. Substrate delivery in this perfusion model does not reflect the physiological fatty acid repertoire sampled by hepatocytes in vivo, as the delivery of the carnitine palmitoyltransferase-independent fat octanoate in the livers of animals maintained in the fed state provokes a convergence of fat oxidation, ketogenic demand, and preexisting glycogen pool sizes not commonly encountered in standard absorptive states. This is likely to overemphasize the differences in vivo in the unaccounted acetyl-CoA balances, and result in variations among relative contributions to HGP, compared with those collected through in vivo studies. Nonetheless, the octanoate perfusion model was employed to specifically reveal the effect of deranged hepatic mitochondrial acetyl-CoA allocation to downstream canonical fates on glucose metabolism, which is likely a primarily lesioned regulatory node in the pathogenesis of $\operatorname{NAFLD}(10,13,18,38)$. Moreover, the portal vein perfusion model specifically quantifies hepatic metabolism and HGP, unlike in vivo approaches that administer tracers via systemic veins and track labeled glucose, which do not exclude the roles of renal or intestinal glucose metabolism.

These experiments revealed an unexpected relationship between hepatic glycogen metabolism and ketogenic insufficiency, and while the unconventional platform of the octanoate-fueled perfusion of a glycogen-loaded liver yielded many of these observations, experiments using livers obtained from mice in the random-fed state also support the impact of ketogenic insufficiency on glycogen metabolism. Hepatic glycogen turnover is primarily controlled by the opposing actions of insulin-stimulated GS, and PYGL (3). Glycogen cycling proceeds from glycogen $\rightarrow \mathrm{G} 1 \mathrm{P} \Leftrightarrow \mathrm{G} 6 \mathrm{P} \Leftrightarrow \mathrm{G} 1 \mathrm{P} \rightarrow \mathrm{UDPG} \rightarrow$ glycogen. Via activating allosteric effects on GS, and inhibitory allosteric effects on PYGL, a regulatory role is served by G6P, which is in equilibrium with 3-carbon gluconeogenic precursors from glycerol and PEP, as well as glucose itself. Livers of ketogenesis-insufficient animals are primed to store glycogen, which may be due in part to reduced abundance of the total pool PYGL, thus diminishing the absolute abundance of active p-PYGL, but other factors may also drive glycogen accumulation. The apparent pool size of G6P was not altered in livers of random-fed ketogenesis-insufficient mice $(m / z$ 259.0224, Supplemental Table 2), limiting the likelihood that variations in G6P concentrations drive the observed differences. During perfusions lacking both insulin and exogenous glucose, the large glycogen pool in livers of ketogenesis-insufficient mice was mobilized, entirely driving the augmentation of hepatic glucose production from livers of ketogenesis-insufficient mice compared with control. Insulin markedly diminished glycogenolysis in both control and ketogenesisinsufficient livers. As GS is the primary insulin-responsive enzyme in hepatic glycogen turnover (39), the normal response to insulin suggests that GS function is likely normal. Nonetheless, despite the normal proportionate response to insulin, glycogenolysis remained elevated in ketogenesis-insufficient livers in the presence of insulin, indicating that mass action or other unidentified allosteric mediators are unique drivers of glycogenolysis in the perfused ketogenesis-insufficient liver.

Livers with engineered ketogenic insufficiency are prone to HFD-induced hepatocellular injury, necrosis, and lobular inflammation. Furthermore, these livers engage de novo lipogenesis from carbohydratederived acetyl-CoA, and have high rates of glycogen turnover that persist in the presence of insulin - all NASH-like signatures, despite the absence of hepatic steatosis and insulin resistance in this model $(19,40)$. In select contexts, such as the neonatal period, livers of ketogenesis-insufficient mice are also steatotic (19). Moreover, despite normal hepatic concentrations within the TAG and DAG classes in the adult chowfed state, untargeted metabolomics and high-resolution lipidomics studies both revealed remodeling of the phospholipidome of livers of ketogenesis-insufficient animals, particularly among numerous species within the BMP lipid class. Strikingly, BMPs are also elevated in steatotic livers from backgrounds that do not exhibit genetically engineered ketogenic insufficiency, but in which ketogenesis is mismatched to hepatocellular lipid delivery. Whether BMP accumulation is a pathogenic, adaptive, or bystander signature will require further investigation. Nonetheless, BMP enrichment in late endosomes and lysosomes suggests engagement of this cellular compartment in all 3 studied models of disordered hepatic lipid metabolism, confirming that organelles beyond lipid droplets and mitochondria should continue to garner investigation 
in lipid metabolism NAFLD/NASH and pathogenesis (41). However, given the absence of overt shifts in hepatic cholesterol homeostasis, BMP species may accumulate outside of normally functioning late endosomes and lysosomes in pathological settings. Synthetic BMP pathways are only partially defined, and mechanisms governing BMP functional properties are unknown. Normal protein abundance of monoacylglycerol lipase ABDH6, an enzyme localized to late endosomes/lysosomes that subsumes $90 \%$ of BMP catabolism, in livers of ketogenesis-insufficient mice also suggests ectopic localization of BMPs within metabolically stressed hepatocytes $(29,30)$. When these results are taken together, it is unclear whether BMP accumulation can be attributed to increased synthesis, decreased clearance, or both. As the observations herein suggest that augmentation of the BMP species was occasionally linked to conditions in which PGs were also modestly expanded, transacylation mechanisms exchanging acyl chains between these 2 pools may be considered in future investigations $(27,28,42)$.

These multiscale observations underscore the need to further develop in humans the relationships among acetyl-CoA homeostasis, TCA cycle flux, glucose metabolism, and their relationships to yet uncharacterized lipid classes in health and disease. Many of these indices can be acquired noninvasively, with or without imaging modalities, and should be considered as measured endpoints in ongoing trials examining pharmacological agents and nutritional/exercise interventions that may mitigate deranged metabolism and fibrosis in the NAFLD/NASH transition $(1,43)$. Moreover, ketogenesis itself may extend beyond a biomarker of NASH progression, and evolve into a target pathway worthy of prosecution. Enthusiasm for therapies that promote ketogenic augmentation, e.g., SGLT2i, is appropriately tempered by concerns for morbidity and mortality of ketoacidosis. Nonetheless, multiple nutritional and pharmacological therapies targeted to myriad disease states are accompanied by, and may be reliant upon, the broad and safe dynamic range exhibited by hepatic ketogenesis. Thus, it is advisable to consider development of novel therapies for metabolic diseases by cautiously accessing the unique properties of the ketogenic pathway while attending to the feared consequence of ketoacidosis.

\section{Methods}

Reagents. All stable isotope-labeled reagents were obtained from Cambridge Isotopes Laboratories, and unless otherwise stated all other chemical reagents and solvents were obtained from Sigma-Aldrich.

Animals. All animal experiments were performed through protocols formally approved by the Institutional Animal Care and Use Committees at Sanford Burnham Prebys Medical Discovery Institute-Lake Nona and the University of Minnesota. Male C57BL/6N $\times$ C57BL/6J mice were maintained on a standard low-fat chow diet, in which $12 \%$ of the calories were from fat, $22 \%$ from protein, and $66 \%$ from carbohydrates (Teklad Global 16\% Protein Rodent Diet) and were given autoclaved water ad libitum. Lights were off between 1800 and 0600 hours in a room maintained at $22^{\circ} \mathrm{C}$. For all adult mouse experiments, mice were housed on paper bedding in groups of 4 to 5 . ASO treatment was initiated in 6-week-old mice by intraperitoneal (i.p.) injection (25 mg/kg) with murine Hmgcs2-targeted ASOs (Ionis, 191229; 5'-CTGTTTGTCACTGCTGGATG) or scrambled sequence control ASOs (Ionis, 141923; 5'-CCTTCCCTGAAGGTTCCTCC) biweekly. For HFD studies, wild-type male C57BL/6N $\times$ C57BL/6J mice were maintained for the indicated number of weeks, starting at 5-6 weeks of age, on a diet in which $60 \%$ of calories were from fat, $20 \%$ from protein, and $20 \%$ from carbohydrates (D12492; Research Diets). Male Lepr $r^{d b} / \operatorname{Lepr}^{d b}(d b / d b)$ mice (Jackson Labs, stock number 000697) were studied at 10 weeks of age.

Portal vein perfusions. Perfusions were performed as previously described (19), and detailed methods are provided in Supplemental Material. Briefly, mice were anesthetized with $50 \mu 1$ of sodium pentobarbital $(65 \mathrm{mg} / \mathrm{ml})$ administered i.p., and once fully unconscious (approximately 5 minutes following delivery), the portal vein was exposed and cannulated with a 24-gauge catheter needle, the abdominal aorta and inferior vena cava cut, and the catheter was firmly tied into the portal vein. The beating heart was then exposed by cutting through the diaphragm and thorax. The right atrium was cut to prevent recirculation of buffer to the liver and to terminate perfusion to the brain. Livers were perfused with our standard oxygenated buffer prewarmed to $42^{\circ} \mathrm{C}$ (determined empirically to counter heat loss in tubing such that tissue delivery is maintained at physiological temperature) with a counter-current heat-exchange circuit and a recirculating water bath. The buffer delivery was maintained at a rate of $8 \mathrm{ml} / \mathrm{min}$ using a peristaltic pump for a period of 1 hour. Perfusate effluent from the initial 30 minutes was discarded, and effluent was collected from the final 30 minutes of the procedure. In addition, 1.8- $\mathrm{ml}$ collections were made at 30, 40, 50 , and 60 minutes after perfusion initiation. 
NMR methods. All NMR resonances were collected using a Bruker Avance III 600 NMR instrument equipped with a CryoProbe. To quantify glucose and $\beta \mathrm{OHB}$ production, and propionate and octanoate consumption, aliquots $(1.8 \mathrm{ml})$ of mouse liver perfusate effluent were collected at 30, 40, 50 and 60 minutes after initiation of the tissue perfusion and stored on ice. These aliquots were frozen following collection and processed for NMR analysis at a later time by thawing, removal of solvent at $45^{\circ} \mathrm{C}$ under vacuum to dryness, dissolving residual solids in $550 \mu$ of $99.9 \%{ }^{2} \mathrm{H}_{2} \mathrm{O}$ spiked with $0.3 \mathrm{mM} \mathrm{d}_{8}$-trimethyl-silyl propionate (TSP), and collecting ${ }^{1} \mathrm{H}-\mathrm{NMR}$ signals under quantitative conditions absent water suppression. Integrated intensities of the $\alpha$-anomeric proton of glucose carbon-1, the methyl signal for $\beta \mathrm{OHB}$, respective $\alpha-\mathrm{CH}_{2}$ signals from propionate and octanoate, as well as the tri-methyl signal from the reference TSP, when corrected for the number of protons, can be used to calculate molar concentrations of the respective substrates. Glucose and $\beta \mathrm{OHB}$ production rates can be calculated from the total effluent recovered over the final 30 minutes of the perfusion along with the liver weight. Glucose and $\beta \mathrm{OHB}$ production rates were calculated as the average from the 4 sample collections. Octanoate consumption can be determined similarly from known initial perfusate concentrations (Supplemental Figure 2A). Acetyl-CoA accounting was performed by assuming that 4 moles of acetyl-CoA are produced from each mole of octanoate consumed. Each mole of $\beta \mathrm{OHB}$ produced consumes 2 moles of acetyl-CoA, while each turn of the TCA cycle disposes 1 mole of acetyl-CoA. For all ${ }^{1} \mathrm{H}-\mathrm{NMR}$ collections, spectra were collected by conventional pulse-and-collect measurements without water suppression and under quantitative conditions (10-ppm spectral range using 15 $\mu$ s $\left[90^{\circ}\right]$ excitation pulse and 22 -second delay between each of 20 transients). NMR methods for glucose sourcing and TCA cycle flux analysis are provided in Supplemental Material.

Glycogen extraction and analysis. Mice were anesthetized with $50 \mu 1$ of sodium pentobarbital (65 mg/ $\mathrm{ml}$ ) administered i.p. Once fully unconscious (approximately 5 minutes following delivery), a transverse incision was made through the skin, fascia, and muscular layers of the lower abdomen, and single lobes of livers were harvested using a liquid nitrogen-chilled Wollenberger-style clamp, and immediately flash-frozen in liquid nitrogen. Frozen liver tissue $(\sim 50 \mathrm{mg})$ was transferred to $0.3 \mathrm{ml}$ of $30 \% \mathrm{KOH} / \mathrm{H}_{2} \mathrm{O}$ solution and maintained at $110^{\circ} \mathrm{C}$ for 30 minutes. Glycogen with salt was precipitated by adding $100 \mu \mathrm{l}$ of $1 \mathrm{M} \mathrm{Na}_{2} \mathrm{SO}_{4}$ and $800 \mu \mathrm{l}$ ethanol and heating to $110^{\circ} \mathrm{C}$ for 5 minutes. A glycogen-rich pellet was obtained by centrifugation at $10,000 \mathrm{~g}$ for 5 minutes. Following decanting the supernatant, the pellet was dissolved in $0.2 \mathrm{ml} \mathrm{H}_{2} \mathrm{O}$ followed by a second precipitation with ethanol and $\mathrm{Na}_{2} \mathrm{SO}_{4}$ and centrifugation. The precipitation was repeated a third time, followed by drying in a SpeedVac (ThermoFisher Scientific). The glycogen was then incubated for 3 hours at $40^{\circ} \mathrm{C}$ in $300 \mu 1$ of a freshly prepared amyloglucosidase solution $(0.3 \mathrm{mg} / \mathrm{ml}$ [30-60 units $/ \mathrm{mg}$ protein] in $0.4 \mathrm{M}$ sodium acetate buffer, $\mathrm{pH} 4.8$ ). Following digestion, the sample was dried under vacuum at $45^{\circ} \mathrm{C}$ and dissolved in $0.55 \mathrm{ml} 0.3 \mathrm{mM}$ TSP in $99.9 \%{ }^{2} \mathrm{H}_{2} \mathrm{O}$. ${ }^{1} \mathrm{H}-\mathrm{NMR}$ spectra were collected under quantitative conditions for the $\alpha$-anomeric proton of glucose carbon-1 at $5.2 \mathrm{ppm}$, and confirmation of glycogen digestion was confirmed by the absence of the glycogen carbon-1 position proton signal at $6.4 \mathrm{ppm}$.

MS methods for untargeted metabolomics. Mice were anesthetized with $50 \mu \mathrm{l}$ of sodium pentobarbital (65 $\mathrm{mg} / \mathrm{ml}$ ) administered i.p. Once fully unconscious (approximately 5 minutes following delivery), a transverse incision was made through the skin, fascia, and muscular layers of the lower abdomen, and single lobes of livers were harvested using a liquid nitrogen-chilled Wollenberger-style clamp, and immediately flash-frozen in liquid nitrogen. Liver was extracted using previously described methods, with modifications (44). Frozen lobules of liver were lyophilized and extracted using cold $\left(-20^{\circ} \mathrm{C}\right) 2: 2: 1$ methanol/acetonitrile $/ \mathrm{H}_{2} \mathrm{O}$ by 3 cycles of vortexing, freeze-thawing, and water bath sonication. The samples were then incubated at $-20^{\circ} \mathrm{C}$ for 1 hour followed by a 10 -minute centrifugation at maximum speed to remove proteins from the solvent. Supernatant was transferred to fresh tubes and evaporated off in the speed-vac. The dried metabolite pellet was reconstituted in $100 \mu 11: 1$ acetonitrile $/ \mathrm{H}_{2} \mathrm{O}$ with the aid of vortexing and sonication, and incubated at $4^{\circ} \mathrm{C}$ for 1 hour to balance solubility of metabolites and prevent any remaining protein from entering into solution. Samples were then centrifuged $\left(10,000 \mathrm{~g}\right.$ for 10 minutes at $\left.4^{\circ} \mathrm{C}\right)$ and the supernatant was used for metabolomics analysis using Phenomenex Luna $\mathrm{NH}_{2}$ column chromatography, and negative ionization mode MS using Thermo Q Exactive Plus with a heated electrospray ionization source. Detailed methods for untargeted metabolomics are provided in Supplemental Material.

MS methods for shotgun lipidomics. Sample preparation for lipids: frozen liver or isolated hepatocytes $(\sim 1 \times$ $10^{6}$ cells) were homogenized in ice-cold water with the aid of 2.8 - and 1.4-mm zirconium oxide beads in a Precellys Evolution homogenizer at $4^{\circ} \mathrm{C}-10^{\circ} \mathrm{C}$. According to the bicinchoninic acid (BCA) protein concentration 
result, an appropriate amount of internal standard mix (containing D14:1 PC, D16:1 PE, D15:0 PG, D14:0 PS, D14:0 PA, N12:0 SM, 14:0 LPE, 17:0 LPC, T14:0 CL, ${ }^{13}$ C4 16:0 carnitine, T17:1 TAG, and 1,3-D15:0 DAG) was added to the homogenate (45). Approximately $130 \mu 1$ of homogenate with internal standard mix was extracted using a previously described method (46), dried under nitrogen, and diluted to a final concentration of approximately $500 \mathrm{fmol} / \mu \mathrm{l}$ lipid. Derivatization and MS were performed using a TSQ Vantage triple quadrupole mass spectrometer as described in detail in Supplemental Material.

Acetyl-CoA measurement. Mice were anesthetized with $50 \mu 1$ of sodium pentobarbital $(65 \mathrm{mg} / \mathrm{ml})$ administered i.p. Once fully unconscious (approximately 5 minutes following delivery), a transverse incision was made through the skin, fascia, and muscular layers of the lower abdomen, and single lobes of livers were harvested using a liquid nitrogen-chilled Wollenberger-style clamp, and immediately flash-frozen in liquid nitrogen. Livers, or isolated mitochondria (see below) stored at $-80^{\circ} \mathrm{C}$ were lyophilized overnight and powdered using a CryoGrinder mortar and pestle (OPS Diagnostics) under liquid nitrogen. Aliquots of powdered liver ( $\sim 5 \mathrm{mg}$ ) were prepared for homogenization in various solvents for organic acid, short-chain CoA, and pyridine nucleotide assays. Acetyl-CoA was quantified using an Agilent 1290 HPLC/6490 triple quadrupole mass spectrometer with electrospray ionization. Calibration standards and liver homogenates were prepared in 5\% cold trichloroacetic acid spiked with stable isotope-labeled internal standards followed by solid-phase extraction (SPE). The methanol extracts from SPE were dried down, reconstituted in HPLC buffer, and CoA was quantified by multiple reaction monitoring using LC-MS/MS.

Mitochondrial isolation. Whole livers were excised, washed in ice-cold PBS, weighed, and suspended in mitochondrial isolation medium (MIM; $300 \mathrm{mM}$ sucrose, $0.2 \mathrm{mM}$ EDTA, $10 \mathrm{mM}$ HEPES, pH 7.4) containing bovine serum albumin (BSA, $1 \mathrm{mg} / \mathrm{ml}$ ). The samples were rapidly homogenized on ice by using a Glas-Col Dounce homogenizer, and centrifuged at $600 \mathrm{~g}$ for 10 minutes at $4^{\circ} \mathrm{C}$. The resulting supernatant, which contained mitochondria, was spun at $8,000 \mathrm{~g}$ for 15 minutes at $4^{\circ} \mathrm{C}$. The resultant supernatant was discarded, the mitochondrial pellet was resuspended in $10 \mathrm{ml}$ of ice-cold MIM-BSA, and the sample centrifuged again at $8,000 \mathrm{~g}$ for 15 minutes at $4^{\circ} \mathrm{C}$. The pellet was briefly washed in ice-cold MIM, protein quantified by BCA assay, and frozen at $-80^{\circ} \mathrm{C}$ prior to lyophilization.

Gene expression analysis. RNA was purified from whole-liver lysates homogenized in RLT Buffer (Qiagen) with 1\% 2-mercaptoethanol using the RNeasy Mini Kit (Qiagen) following the manufacturer's guidelines. Reverse transcripts were generated using SuperScript II (Invitrogen), while real-time reverse-transcription PCR (RT-PCR) was performed using SsoAdvanced Universal SYBR Green Supermix (Bio-Rad) on a CFX384 Real-Time System (Bio-Rad). Transcripts were quantified using the $2^{-\Delta \Delta \mathrm{Ct}}$ method, with $R p l 32$ as an internal reference. Primer sequences were as follows: Tamm41, forward 5'-TGGAGCCACTACTCCTTCCT, reverse 5'-TGATAAGCTTCCCGTCACACC; Pgs1, forward 5'-AGGTTGTGGATGGGATGGTGCA, reverse 5'-TCATGGACGTCATCCACTCG; Crls1, forward 5'-CGCCAGCTCGTATGAAAATCC, reverse 5'-AATCAGGTAGCCCAACACGG; Ptpmt1, forward 5'-ACACCTCGAAGGAATGGAAGAA, reverse 5'-CTTGAGAGCAAACTGGACTCCT. All abundances were normalized to Rpl32: forward 5'-CCTCTGGTGAAGCCCAAGATC, reverse 5'-TCTGGGTTTCCGCCAGTTT.

Isolation of mouse primary hepatocytes. Mice were anesthetized with pentobarbital, the portal vein was cannulated, and the liver was perfused first with solution $1\left(\mathrm{Ca}^{+2} / \mathrm{Mg}^{+2}\right.$-free HEPES solution containing 0.5 mM EGTA) for 5 minutes and then with solution $2\left(\mathrm{Ca}^{+2} / \mathrm{Mg}^{+2}\right.$-free $\mathrm{HEPES}$ containing $5 \mathrm{mM} \mathrm{CaCl}_{2} \cdot \mathrm{H}_{2} \mathrm{O}$, and type IV collagenase, 0.5-5.0 FALGPA units/mg solid, $\geq 125 \mathrm{CDU} / \mathrm{mg}$ solid) for 10 minutes. Liver lobes were submerged in solution 2 and left for an additional 4 minutes at room temperature. Collagenase activity was terminated by addition of $10 \mathrm{ml}$ cold DMEM. Tissue was manually dissociated with forceps, filtered with $70-\mu \mathrm{m}$ cell strainers, and spun at $50 \mathrm{~g}$ for 2 minutes at $4^{\circ} \mathrm{C}$. Pelleted cells were washed twice with DMEM/10\%FBS followed by centrifugation at $50 \mathrm{~g}$ for 2 minutes at $4^{\circ} \mathrm{C}$. The cell suspension was layered on a Percoll cushion (prepared by mixing $10 \mathrm{ml}$ of cold Percoll stock with $10 \mathrm{ml}$ of cold medium) and centrifuged at $160 \mathrm{~g}$ at for 5 minutes at $4^{\circ} \mathrm{C}$. The viable hepatocyte pellet was resuspended in $20 \mathrm{ml}$ of $\mathrm{DMEM} / 10 \% \mathrm{FBS}$ and spun at $50 \mathrm{~g}$ for 2 minutes at $4^{\circ} \mathrm{C}$. Cells were resuspended in cold DMEM/10\% FBS and viability was confirmed by trypan blue. Isolated hepatocytes $\left(\sim 1 \times 10^{6}\right.$ cells $)$ were left at $-80^{\circ} \mathrm{C}$ until used for lipidomics analysis.

Total hepatic cholesterol concentrations. Livers from mice were homogenized in water $(5: 1, \mathrm{v} / \mathrm{g})$ with 2.8- and 1.4-mm zirconium oxide beads in a Precellys Evolution homogenizer at $4^{\circ} \mathrm{C}-10^{\circ} \mathrm{C}$. Cholesterol was extracted with $\mathrm{CHCl}_{3} / \mathrm{CH}_{3} \mathrm{OH}$ solution and centrifuged $\left(5,000 \mathrm{~g}, 5\right.$ minutes, $\left.4^{\circ} \mathrm{C}\right)$. The organic phase was recovered, extracted with chloroform, centrifuged $\left(5,000 \mathrm{~g}, 5\right.$ minutes, $\left.4^{\circ} \mathrm{C}\right)$ and evaporated using a 
SpeedVac. Dried samples were resuspended in a kit-supplied buffer (Wako Diagnostics, Cholesterol E kit) and analyzed according to the manufacturer's instructions.

Immunoblotting. Protein extracts were collected from approximately $50 \mathrm{mg}$ frozen liver samples in 10 times volume protein lysis buffer containing $20 \mathrm{mM}$ Tris- $\mathrm{HCl}, 150 \mathrm{mM} \mathrm{NaCl}, 1 \mathrm{mM}$ EDTA, and $1 \%$ Triton $\mathrm{X}-100$ at $\mathrm{pH} 7.5$ and supplemented with protease inhibitor cocktail (complete mini EDTA-free, Roche) and phosphatase inhibitor cocktail (Sigma-Aldrich, 524629). Immunoblot analysis was performed as described previously (20). All blots were blocked with 5\% milk/PBST (PBS/0.01\% Tween-20). The phosphorylated form of the liver-specific isoform glycogen phosphorylase (p-PYGL) was specifically detected using a sheep antibody raised against human glycogen phosphorylase $b$ (PYGL)-phosphoserine 15 (acquired from MRC PPU University of Dundee, S961A; 1:500 in 5\% BSA/ PBST). Total PYGL was detected using a rabbit antibody raised against PYGL (St. John's Laboratory, ABIN2564827; 1:2,000 in 5\% milk/PBST). p-GS was detected using a rabbit antibody raised against pan-GS [Cell Signaling Technology, Phospho-Glycogen Synthase (Ser641) (D4H1B) XP Rabbit mAb $47043 ; 1: 2,000$ in 5\% BSA/PBST]. Total GS was detected using a rabbit antibody raised against pan-GS [Cell Signaling Technology, Glycogen Synthase (15B1) Rabbit mAb 3886); 1:1,000 in 5\% milk/PBST]. HMGCS2 protein was detected using a rabbit polyclonal from Santa Cruz Biotechnology [mHMGCS Antibody (H-70), sc-33828; 1:1,000 in in 5\% milk/PBST]. ABHD6 protein was detected using rabbit polyclonal anti-ABDH6 (abcam 133855; 1:2,000). Actin normalizing controls were blotted using a rabbit polyclonal anti-actin (Sigma-Aldrich, A2066; 1:1,000 in in 5\% milk/PBST) Secondary antibodies were all obtained as goat anti-rabbit IgG (Southern Biotech; 1:15,000) conjugated to horseradish peroxidase (HRP), with the exception of p-PYGL, which was a mouse anti-sheep HRP conjugate, Jackson Immunoresearch, 213-032-177; 1:10,000 in 5\% milk/PBST). Band intensities were measured densitometrically using Quantity One software (Bio-Rad).

Serum ketone measurement. Total ketone bodies, $\beta \mathrm{OHB}$, and AcAc were quantified using standard biochemical assays coupled to colorimetric substrates (Wako).

Histology. For hematoxylin and eosin-stained sections, fixed, paraffin-embedded liver was microtomesectioned, stained and photographed using standard methods.

Statistics. Analyses were performed with GraphPad Prism software. Unpaired 2-tailed Student's $t$ tests, or 2- or 1-way ANOVA, with corrections for multiple comparisons, were used as appropriate, as indicated within the text and figure legends. Data are presented as the means \pm SEM unless otherwise indicated. A $P$ value of less than 0.05 was considered significant.

Study approval. All animal experiments were performed through protocols formally approved by the Institutional Animal Care and Use Committees at Sanford Burnham Prebys Medical Discovery InstituteLake Nona and the University of Minnesota.

\section{Author contributions}

$\mathrm{DAD}, \mathrm{PP}, \mathrm{BE}$, and PAC designed the research studies. DAD, PP, BE, YJC, and SEM conducted experiments and acquired data. DAD, PP, YJC, SEM, XH, GJP, and PAC analyzed the data. PAC and MJG provided reagents. DAD, PP, BE, YJC, SEM, MJG, GJP, XH, and PAC wrote the manuscript.

\section{Acknowledgments}

The authors thank J. Matthew Gandy for expert portal vein perfusions, Matthew Longo for technical support, Stephen Gardell and Christopher Petucci for LC-MS/MS acetyl-CoA quantification, Peter E. Phelan for advice on primary hepatocyte isolation, Miao Wang for advice on development of the measurement of BMPs, and Laura Kyro for graphics expertise. This work was supported in part by a grant from the NIH (DK091538 to P.A. Crawford).

Address correspondence to: Peter A. Crawford, University of Minnesota, 420 Delaware St SE, MMC 605, Minneapolis, Minnesota 55455, USA. Phone: 612.301.2202; Email: crawforp@umn.edu.

1. Diehl AM, Day C. Cause, pathogenesis, and treatment of nonalcoholic steatohepatitis. NEngl J Med. 2017;377(21):2063-2072.

2. Samuel VT, Shulman GI. Nonalcoholic fatty liver disease as a nexus of metabolic and hepatic diseases. Cell Metab. 2018;27(1):22-41.

3. Samuel VT, Shulman GI. The pathogenesis of insulin resistance: integrating signaling pathways and substrate flux. $J$ Clin Invest. 
2016;126(1):12-22.

4. Rinella ME, Sanyal AJ. Management of NAFLD: a stage-based approach. Nat Rev Gastroenterol Hepatol. 2016;13(4):196-205.

5. Hardy T, Oakley F, Anstee QM, Day CP. Nonalcoholic fatty liver disease: pathogenesis and disease spectrum. Annu Rev Pathol. 2016;11:451-496.

6. Puchalska P, Crawford PA. Multi-dimensional roles of ketone bodies in fuel metabolism, signaling, and therapeutics. Cell Metab. 2017;25(2):262-284.

7. Cox PJ, et al. Nutritional ketosis alters fuel preference and thereby endurance performance in athletes. Cell Metab. 2016;24(2):256-268.

8. Elia M, Wood S, Khan K, Pullicino E. Ketone body metabolism in lean male adults during short-term starvation, with particular reference to forearm muscle metabolism. Clin Sci. 1990;78(6):579-584.

9. Balasse EO, Fery F, Neef MA. Changes induced by exercise in rates of turnover and oxidation of ketone bodies in fasting man. J Appl Physiol Respir Environ Exerc Physiol. 1978;44(1):5-11.

10. Satapati S, et al. Mitochondrial metabolism mediates oxidative stress and inflammation in fatty liver. J Clin Invest. 2015;125(12):4447-4462.

11. Koliaki C, et al. Adaptation of hepatic mitochondrial function in humans with non-alcoholic fatty liver is lost in steatohepatitis. Cell Metab. 2015;21(5):739-746.

12. Satapati S, et al. Elevated TCA cycle function in the pathology of diet-induced hepatic insulin resistance and fatty liver. $J$ Lipid Res. 2012;53(6):1080-1092.

13. Sunny NE, Parks EJ, Browning JD, Burgess SC. Excessive hepatic mitochondrial TCA cycle and gluconeogenesis in humans with nonalcoholic fatty liver disease. Cell Metab. 2011;14(6):804-810.

14. Bickerton AS, et al. Adipose tissue fatty acid metabolism in insulin-resistant men. Diabetologia. 2008;51(8):1466-1474.

15. Vice E, Privette JD, Hickner RC, Barakat HA. Ketone body metabolism in lean and obese women. Metab Clin Exp. 2005;54(11):1542-1545.

16. Soeters MR, et al. Effects of insulin on ketogenesis following fasting in lean and obese men. Obesity (Silver Spring). 2009;17(7):1326-1331

17. Bergman BC, Cornier MA, Horton TJ, Bessesen DH. Effects of fasting on insulin action and glucose kinetics in lean and obese men and women. Am J Physiol Endocrinol Metab. 2007;293(4):E1103-E1111.

18. Sunny NE, et al. Progressive adaptation of hepatic ketogenesis in mice fed a high-fat diet. Am J Physiol Endocrinol Metab. 2010;298(6):E1226-E1235.

19. Cotter DG, et al. Ketogenesis prevents diet-induced fatty liver injury and hyperglycemia. J Clin Invest. 2014;124(12):5175-5190

20. Wentz AE, et al. Adaptation of myocardial substrate metabolism to a ketogenic nutrient environment. J Biol Chem. 2010;285(32):24447-24456.

21. Schugar RC, Moll AR, André d'Avignon D, Weinheimer CJ, Kovacs A, Crawford PA. Cardiomyocyte-specific deficiency of ketone body metabolism promotes accelerated pathological remodeling. Mol Metab. 2014;3(7):754-769.

22. Jin ES, Jones JG, Merritt M, Burgess SC, Malloy CR, Sherry AD. Glucose production, gluconeogenesis, and hepatic tricarboxylic acid cycle fluxes measured by nuclear magnetic resonance analysis of a single glucose derivative. Anal Biochem. 2004;327(2):149-155.

23. Mayorek N, Bar-Tana J. Medium chain fatty acids as specific substrates for diglyceride acyltransferase in cultured hepatocytes. J Biol Chem. 1983;258(11):6789-6792.

24. Previs SF, Kelley DE. Tracer-based assessments of hepatic anaplerotic and TCA cycle flux: practicality, stoichiometry, and hidden assumptions. Am J Physiol Endocrinol Metab. 2015;309(8):E727-E735.

25. Huang X, Chen YJ, Cho K, Nikolskiy I, Crawford PA, Patti GJ. X13CMS: global tracking of isotopic labels in untargeted metabolomics. Anal Chem. 2014;86(3):1632-1639.

26. Yao $\mathrm{CH}$, et al. Exogenous fatty acids are the preferred source of membrane lipids in proliferating fibroblasts. Cell Chem Biol. 2016;23(4):483-493

27. Gallala HD, Sandhoff K. Biological function of the cellular lipid BMP-BMP as a key activator for cholesterol sorting and membrane digestion. Neurochem Res. 2011;36(9):1594-1600.

28. Hullin-Matsuda F, Luquain-Costaz C, Bouvier J, Delton-Vandenbroucke I. Bis(monoacylglycero)phosphate, a peculiar phospholipid to control the fate of cholesterol: Implications in pathology. Prostaglandins Leukot Essent Fatty Acids. 2009;81(5-6):313-324.

29. Thomas G, et al. The serine hydrolase ABHD6 is a critical regulator of the metabolic syndrome. Cell Rep. 2013;5(2):508-520.

30. Pribasnig MA, et al. $\alpha / \beta$ Hydrolase domain-containing 6 (ABHD6) degrades the late endosomal/lysosomal lipid bis(monoacylglycero)phosphate. J Biol Chem. 2015;290(50):29869-29881.

31. Wang Z, et al. Specific metabolic rates of major organs and tissues across adulthood: evaluation by mechanistic model of resting energy expenditure. Am J Clin Nutr. 2010;92(6):1369-1377.

32. Perry RJ, et al. Propionate increases hepatic pyruvate cycling and anaplerosis and alters mitochondrial metabolism. J Biol Chem. 2016;291(23):12161-12170.

33. Hasenour CM, et al. Mass spectrometry-based microassay of (2)H and (13)C plasma glucose labeling to quantify liver metabolic fluxes in vivo. Am J Physiol Endocrinol Metab. 2015;309(2):E191-E203.

34. Hughey CC, et al. Loss of hepatic AMP-activated protein kinase impedes the rate of glycogenolysis but not gluconeogenic fluxes in exercising mice. J Biol Chem. 2017;292(49):20125-20140.

35. Martinez R, et al. Endogenous glucose production and hormonal changes in response to canagliflozin and liraglutide combination therapy. Diabetes. 2018;67(6):1182-1189.

36. Zinman B, et al. Empagliflozin, cardiovascular outcomes, and mortality in type 2 diabetes. N Engl J Med. 2015;373(22):2117-2128.

37. Neal B, et al. Canagliflozin and cardiovascular and renal events in type 2 diabetes. NEngl J Med. 2017;377(7):644-657.

38. Perry RJ, Peng L, Cline GW, Petersen KF, Shulman GI. A non-invasive method to assess hepatic acetyl-CoA in vivo. Cell Metab. 2017;25(3):749-756.

39. Petersen KF, Laurent D, Rothman DL, Cline GW, Shulman GI. Mechanism by which glucose and insulin inhibit net hepatic glycogenolysis in humans. J Clin Invest. 1998;101(6):1203-1209. 
40. Lambert JE, Ramos-Roman MA, Browning JD, Parks EJ. Increased de novo lipogenesis is a distinct characteristic of individuals with nonalcoholic fatty liver disease. Gastroenterology. 2014;146(3):726-735.

41. Madrigal-Matute J, Cuervo AM. Regulation of liver metabolism by autophagy. Gastroenterology. 2016;150(2):328-339.

42. Hullin-Matsuda F, et al. De novo biosynthesis of the late endosome lipid, bis(monoacylglycero)phosphate. J Lipid Res. 2007;48(9):1997-2008.

43. Musso G, Cassader M, Gambino R. Non-alcoholic steatohepatitis: emerging molecular targets and therapeutic strategies. Nat Rev Drug Discov. 2016;15(4):249-274

44. Chen YJ, et al. Lactate metabolism is associated with mammalian mitochondria. Nat Chem Biol. 2016;12(11):937-943.

45. Han X, Yang J, Cheng H, Ye H, Gross RW. Toward fingerprinting cellular lipidomes directly from biological samples by twodimensional electrospray ionization mass spectrometry. Anal Biochem. 2004;330(2):317-331.

46. Wang C, Wang M, Zhou Y, Dupree JL, Han X. Alterations in mouse brain lipidome after disruption of CST gene: a lipidomics study. Mol Neurobiol. 2014;50(1):88-96. 